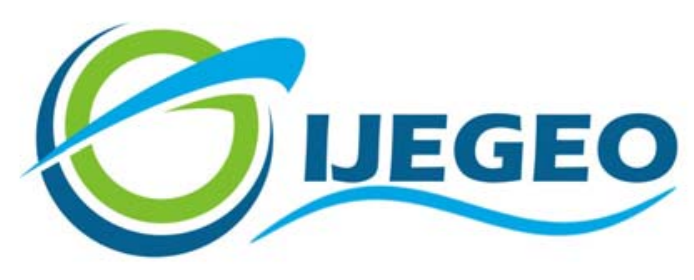

International Journal of Environment and Geoinformatics (IJEGEO) is an international, multidisciplinary, peer reviewed, open access journal.

\title{
Remote sensing approaches and mapping methods for monitoring soil salinity under different climate regimes
}

\section{Taha GORJI, Aylin YILDIRIM, Elif SERTEL \& Aysegül TANIK}

\author{
Chief in Editor \\ Prof. Dr. Cem Gazioğlu \\ Co-Editor \\ Prof. Dr. Dursun Zafer Şeker, Prof. Dr. Şinasi Kaya, \\ Prof. Dr. Ayşegül Tanık and Assist. Prof. Dr. Volkan Demir
}

Editorial Committee (2019)

Assos. Prof. Dr. Abdullah Aksu (TR), Prof. Dr. Bedri Alpar (TR), Prof. Dr. Lale Balas (TR), Prof. Dr. Levent Bat (TR), Prof. Dr. Paul Bates (UK), Prof. Dr. Bülent Bayram (TR), Prof. Dr. Luis M. Botana (ES), Prof. Dr. Nuray Çağlar (TR), Prof. Dr. Sukanta Dash (IN), Dr. Soofia T. Elias (UK), Prof. Dr. A. Evren Erginal (TR), Assoc. Prof. Dr. Cüneyt Erenoğlu (TR), Dr. Dieter Fritsch (DE), Assos. Prof. Dr. Çiğdem Göksel (TR), Prof.Dr. Lena Halounova (CZ), Dr. Hakan Kaya (TR), Assoc. Prof. Dr. Maged Marghany (MY), Prof. Dr. Michael Meadows (ZA), Prof. Dr. Nebiye Musaoğlu (TR), Prof. Dr. Erhan Mutlu (TR), Prof. Dr. Masafumi Nakagawa (JP), Prof. Dr. Hasan Özdemir (TR), Prof.Dr. Chryssy Potsiou (GR), Prof. Dr. Erol Sarı (TR), Prof. Dr. Maria Paradiso (IT), Prof. Dr. Petros Patias (GR), Prof. Dr. Elif Sertel (TR), Prof. Dr. Nüket Sivri (TR), Assoc. Prof. Dr. Füsun Balık Şanlı (TR), Prof. Dr. Uğur Şanlı (TR), Assoc. Prof. Dr. Oral Yağcı (US), Prof. Dr. Seyfettin Taş (TR), Assoc. Prof. Dr. Ömer Suat Taşkın (TR), Dr. İnese Varna (LV), Dr. Petra Visser (NL), Prof. Dr. Selma Ünlü (TR), Assoc. Prof. Dr. İ. Noyan Y1lmaz (AU), Prof. Dr. Murat Yakar (TR), Assit. Prof. Dr. Sibel Zeki (TR) 


\title{
Remote sensing approaches and mapping methods for monitoring soil salinity under different climate regimes
}

\author{
Taha Gorji $^{1}$ iD, Aylin Yıldırım² $^{\text {ID, }}$ Elif Sertel $^{3}$ (D), Aysegül Tanık ${ }^{4, *}$ iD \\ ${ }^{1}$ Informatics Institute, Geographical Information Technology Program, Istanbul Technical University (ITU), 34469, Maslak-Istanbul/TR \\ ${ }_{2}^{2}$ Informatics Institute, Satellite Communication and Remote Sensing Program, Istanbul Technical University (ITU), 34469, Maslak-Istanbul/TR \\ ${ }^{3}$ Faculty of Civil Engineering, Department of Geomatics Engineering, Istanbul Technical University (ITU), 34469, Maslak-Istanbul/TR \\ ${ }^{4}$ Faculty of Civil Engineering, Department of Environmental Engineering. Istanbul Technical University (ITU), 34469, Maslak-Istanbul/TR
}

How to cite: Gorji et al. (2019). Remote sensing approaches and mapping methods for monitoring soil salinity under different climate regimes, International Journal of Environment and Geoinformatics (IJEGEO), 6(1): 33-49. DOI: 10.30897/ijegeo.500452

\begin{abstract}
Soil salinization is one of the severe land-degradation problems due to its adverse effects on land productivity. Each year several hectares of lands are degraded due to primary or secondary soil salinization, and as a result, it is becoming a major economic and environmental concern in different countries. Spatio-temporal mapping of soil salinity is therefore important to support decisionmaking procedures for lessening adverse effects of land degradation due to the salinization. In that sense, satellite-based technologies provide cost effective, fast, qualitative and quantitative spatial information on saline soils.

The main objective of this work is to highlight the recent remote sensing (RS) data and methods to assess soil salinity that is a worldwide problem. In addition, this study indicates potential linkages between salt-affected land and the prevailing climatic conditions of the case study areas being examined. Web of science engine is used for selecting relevant articles. "Soil salinity" is used as the main keyword for finding "articles" that are published from January 1, 2007 up to April 30, 2018. Then, 3 keywords; "remote sensing", "satellite" and "aerial" were used to filter the articles. After that, 100 case studies from 27 different countries were selected. Remote sensing based researches were further overviewed regarding to their location, spatial extent, climate regime, remotely sensed data type, mapping methods, sensing approaches together with the reason of salinity for each case study. In addition, soil salinity mapping methods were examined to present the development of different RS based methods with time. Studies are shown on the Köppen-Geiger climate classification map. Analysis of the map illustrates that $63 \%$ of the selected case study areas belong to arid and semi-arid regions. This finding corresponds to soil characteristics of arid regions that are more susceptible to salinization due to extreme temperature, high evaporation rates and low precipitation.
\end{abstract}

Keywords: Soil Salinity, Remote Sensing, Mapping Methods, Sensing Approaches, Arid environment.

\section{Introduction}

Soils play a significant role in understanding and solving the worldwide environmental issues including climate change effects, food and water security, land degradation and impact of habitat loss on species (Arrouays et al., 2017; İmamoğlu \& Sertel, 2016; Gazioğlu et al., 2010). Therefore, assessment of soil properties like soil salinity is crucial for land sustainability at local and regional scale (Grunwald et al., 2015). In arid and semi-arid regions of the world, soil salinization is one of the main drastic phenomena due to its adverse effects on land productivity and plant growth. As population is increasing rapidly, demand for supplying food is rising; however, plenty of cultivated land is deserted because of primary and secondary soil salinization. Salt expansion in soil due to natural processes containing physical or chemical weathering and its movement from parent material, geological deposits or groundwater is known as primary salinization. On the contrary, secondary salinization is introduced by human interventions (Barut, 2015; Daliakopoulos et al., 2016; Kaya et al., 2017;
Gazioğlu, 2018; Esetlili et al., 2018). On the other hand, traditional irrigation methods and inadequate drainage systems can be addressed as the two main humaninduced reason of secondary salinization (Gorji et al., 2017) that adversely impact almost $20 \%$ of irrigated land worldwide (Mayak et al., 2004). Metternicht and Zinck (2003) highlighted that around 955 million ha of land in the world is salinized due to primary salinization, and approximately 77 million ha is affected because of secondary salinization.

Soil salinity is among the common soil characteristics that effects agricultural production, and it causes severe worldwide environmental problems particularly in arid and semi-arid areas. In these regions, precipitation is inadequate to keep natural percolation of water within the soil profile, and thus, it leads to accumulation of soluble salts in soil with negative effect on soil structure (Mulder et al., 2011; Ülker et al., 2018). It is considered that nearly all continents of the world are facing soil salinization problem, albeit dry regions are naturally more affected than humid ones due to low precipitation 
rate and high evaporation which leads to limited leaching of soluble salts through the soil profile (Zinck \& Metternicht., 2008). Matinfar et al. (2013) underlined that especially traditional irrigation methods exacerbate soil salinization and deteriorate soil quality, and correspondingly destructive impacts of soil salinization on seed germination restrict plant growth. In addition, saline soil prevents water intake to plants from root zone due to diminishing osmotic potential of soil water (Bhatt et al., 2008). Increasing demand for food supply due to rapid population growth will result in more change of dry lands to agricultural land in future, and as a consequence, salinization hazard will extend due to irrigation. Therefore, temporal monitoring and assessment of saline soil is highly important for reducing its adverse effects like land degradation and diminishing crop yields (Allbed \& Kumar, 2013).

Traditional field-based soil salinity measurement methods constrain particular and continuous monitoring of saline soils since those methods are limited in time and space, and can only provide point-wise information. Spatio-temporal mapping of soil salinity is significant to support decision-making procedures for diminishing adverse effects of soil salinization. In that sense, satellite-based technologies provide cost-effective, fast, qualitative and quantitative spatial information on saline soils (Gorji et al., 2017a; Gorji et al., 2018). Remotely sensed data is an efficient data source to produce variety of salt-affected soil maps in conjunction with field measurements. As such, soil analysis by utilizing modern technological tools of Remote Sensing (RS) and Geographical Information System (GIS) provides a valuable resource inventory related to the well-being of land especially those allocated for agricultural production (Manchanda et al., 2002). RS tools such as aerial photography, videography, infrared thermometry, RADAR and LIDAR imagery and especially multispectral scanners, have contributed remarkable development of algorithms and models for mapping and assessing of soil salinity (Abbas et al., 2013a; Gorji et al., 2017b; Büyüksalih \& Gazioğlu, 2019). Utilizing multispectral RS data has considerably improved monitoring of soil salinity; however, transformation procedure is required prior to accurate change detection since identifying changes in two different periods of time include uncertainties in terms of probability, nature, and magnitude of the variations, and it can be recognized using expert knowledge as pointed out by Metternicht \& Zinck (2003). Hyperspectral RS data is another widely used source for soil salinity detection since considerable amount of information can be extracted due to high spatial and spectral resolution of these data (Justin and Suresh, 2015). Specifically, hyperspectral imagery can contribute to accurate soil salinity mapping in regions with vegetation cover of salt tolerant plants because of its capability to differ halophytic plant cover from nonhalophytes. Knowledge of understanding salinity impacts on spectral reflectance of both soil and vegetation is a primary key for utilizing hyperspectral imagery to map saline regions (Dutkiewicz, 2006). Pixel-by-pixel basis detection of spatial variation in soil salinity can be performed also by using airborne remote sensing. This possibility has high significance particularly for faraway and remote regions; since it can contribute information on environmental changes (Goldshleger et al., 2010). One of the main restrictions of using RS data for mapping salinity is linked with high vertical, spatial and temporal changes of salinity in different layers of the soil. Despite, remotely sensed data cannot provide information from entire soil profile due to observing only the surface area (Mulder et al., 2011). Spectral response behavior of salt-affected soil is the primary factor for acquiring information from RS data. Visible and near-infrared spectral bands are more sensitive to detecting saline soil since there is a significance reflectance in these bands in comparison with spectral reflectance of soil in a farmland unaffected by salinization (Ding et al., 2011; Gorji et al., 2015). Several parameters including salt content, soil moisture content, color and texture affect spectral reflectance of soil. As such, RS tools can provide information on the presence of salinity directly on barren land and indirectly on vegetated areas according to the characteristics of vegetation. Various salinity and vegetation indices have been created by combination of spectral bands for detecting salt-affected regions, and they have been examined with different outcomes. Indeed, selecting and utilizing a specific index may not achieve the best result in all cases, since level of salinity and extent of vegetation cover is different for each case study (Allbed and Kumar, 2013). Acquiring accurate result for mapping soil salinity via RS requires appropriate timing for fieldwork and measurement. Obtaining RS data in parallel to field survey is necessary for checking the validation of the results. In addition, as salt accumulation in the soil is season-specific; a dry season is more suitable for conducting soil salinity studies since in rainy season high amount of precipitation may lead to washing surface salt and diminishing the salinity level in the topsoil (Shrestha \& Farshad, 2009).

This study summarizes the trends and advances of RS technology for soil salinity monitoring and mapping within the past decade through analyzing 100 case studies from 27 different countries for scientists, engineers and practitioners with the aim of facilitating future studies and researches. In addition, it indicates potential linkage between salt-affected land and the prevailing climatic conditions of the case study areas being examined.

\section{Methodology used}

Initially "soil salinity" was used as the main keyword for finding only "articles" that were published in Web of Science (WoS) from January 1, 2007 up to April 30, 2018. Totally, 2702 results were found on utilizing RS techniques for mapping soil salinity in various geographical locations, and is clearly seen that this subject is becoming more common as monitoring soil salinity is the essential step to diminish its adverse effects. In the next phase, 3 keywords namely "remote sensing", "satellite" and "aerial" were added for filtering the results and linking soil salinity to RS technology and all analysis algorithms therein. As illustrated in the flow 
diagram of the methodology used (Figure 1), number of articles that were found for "remote sensing", "satellite" and "aerial" keywords are 163, 83 and 32, respectively. After screening and reviewing the results, 100 case studies on RS approaches and mapping methods for monitoring soil salinity were selected. According to the screening criteria applied to select the appropriate articles that fit to the objective of this review article, all the case studies that monitor a specific region and assess salinity condition of the area were selected, and those researches that theoretically examine saline soil were excluded. Selected case studies utilized satellite images or aerial photographs and applied RS mapping methods. Those researches that conducted proximal sensing or implemented only geo-statistical approaches are eliminated. Studies that used soil salinity as a secondary variable for other applications were also excluded from this study. RS based researches were further evaluated regarding to their spatial extent, climate regime, satellite data, mapping methods, sensing approaches and reason of salinity for the selected case studies. Spatial extent of each case study was classified under local or regional categories as well indicating its surface area. Climate regime of the studies was labeled based on KöppenGeiger climate classification. Climatic combinations described with the Köppen-Geiger classification are ecologically suitable and geographic distribution of the long-term mean climate and associated ecosystem circumstances are mapped by utilizing this climate classification. The classification categorizes climate by a number of specific seasonal, temperature and precipitation regimes based on the combination of seasonal temperature and precipitation values (Chen \& Chen, 2013).

Figure 2 is prepared as a summary of the methodology used in the form of clusters. It shows the overall number of selected articles and their interaction with each other. It is clearly observed that the majority of the selected articles are found to be based on "remote sensing" keyword. In addition, 37 articles are selected for both "remote sensing" and "satellite images" keywords. Among the results found under "aerial photograph" keyword, only 3 fitted to the objective of this article. Out of these 3 selected articles, 2 were similarly found by using all 3 keywords and the other one was a common result for keywords "aerial photograph" and "satellite images". Distribution of the number of published WoS articles that comply with the objective of this review work is shown in Figure 3 where the increasing trend of monitoring soil salinity via RS in the recent years is clearly seen.

Moreover, satellite data and RS mapping algorithms were summarized for each case study to illustrate the trends of RS application for monitoring soil salinity within years. Sensing approaches were categorized according to methods and tools used for obtaining information on saline soil including satellite images, airborne photogrammetry, field measurement and laboratory analysis. In addition, this review work stated natural or human-induced causes of soil salinization in each case study. Studies were further sorted based on their publication year for achieving the developments of RS methods that were used for monitoring and detection of soil salinity within the last decade. A matrix was set up as shown in Table 1 where published year of each selected article together with the study area, spatial extent, climate regime, satellite data, mapping methods, sensing approaches and reason of salinity in each study are the parameters which formed the columns and the information for each case study was sorted according to time in the form of rows. This matrix includes a total of 100 case studies from 27 countries.

The novelty of the work lies on the matrix that was specifically formed for this study by focusing on RS technology as well as on categorizing the case study areas according to their climate regime by plotting each on the Köppen-Geiger climate classification map. Soil scientists, practitioners, engineers, scientists, land planner, decision and policy makers as well as the young researchers could benefit from the compact information compiled in this matrix (Table 1).

\section{Results and Discussion}

Increasing number of studies on detecting and monitoring soil salinity indicates the common interest towards preserving soil fertility and lessening adverse effects of salinization. Specific findings including shared and new mapping techniques, sensing approaches, satellite data and reason of salinity for each case study are summarized in Table 1 . This review work presents that majority of the recent studies preferred to utilize RS technology in comparison to other tools for monitoring soil salinity. Advantages of RS technology in terms of cost, time and manpower that is required for completing a case study outperformed other traditional methods. It is also indicated that minimum field survey and sampling is required for studies that are conducted based on RS technology, since it is necessary to validate RS data with ground truth measurements.

Summary of the selected case studies are sorted based on their publication years from the most recent to the oldest to illustrate the advancement and development of RS algorithms and techniques with time. Some common approaches like utilizing ordinary salinity and vegetation indices, correlation and regression analysis, principal component analysis (PCA), decision tree classification (DTC), partial least square regression (PLSR), maximum likelihood classification have been widely used in the past and still they are also favored in the recent studies. Especially after year 2011, new salinity indices similar to canopy response salinity index are derived from a combination of spectral bands, and this tendency of innovating new soil salinity and vegetation indices is still on-going. Moreover, application of some models and classification techniques such as support vector machine (SVM), random forest (RF) regression models, neural network model and some other new generated models have been broadly in use in the recent years. This overview points out that selecting appropriate soil salinity mapping approach for each case study is rather based on the availability of data and on the specific condition of that study area; and more importantly, it is crystal clear that there is no best method that can be examined globally. 


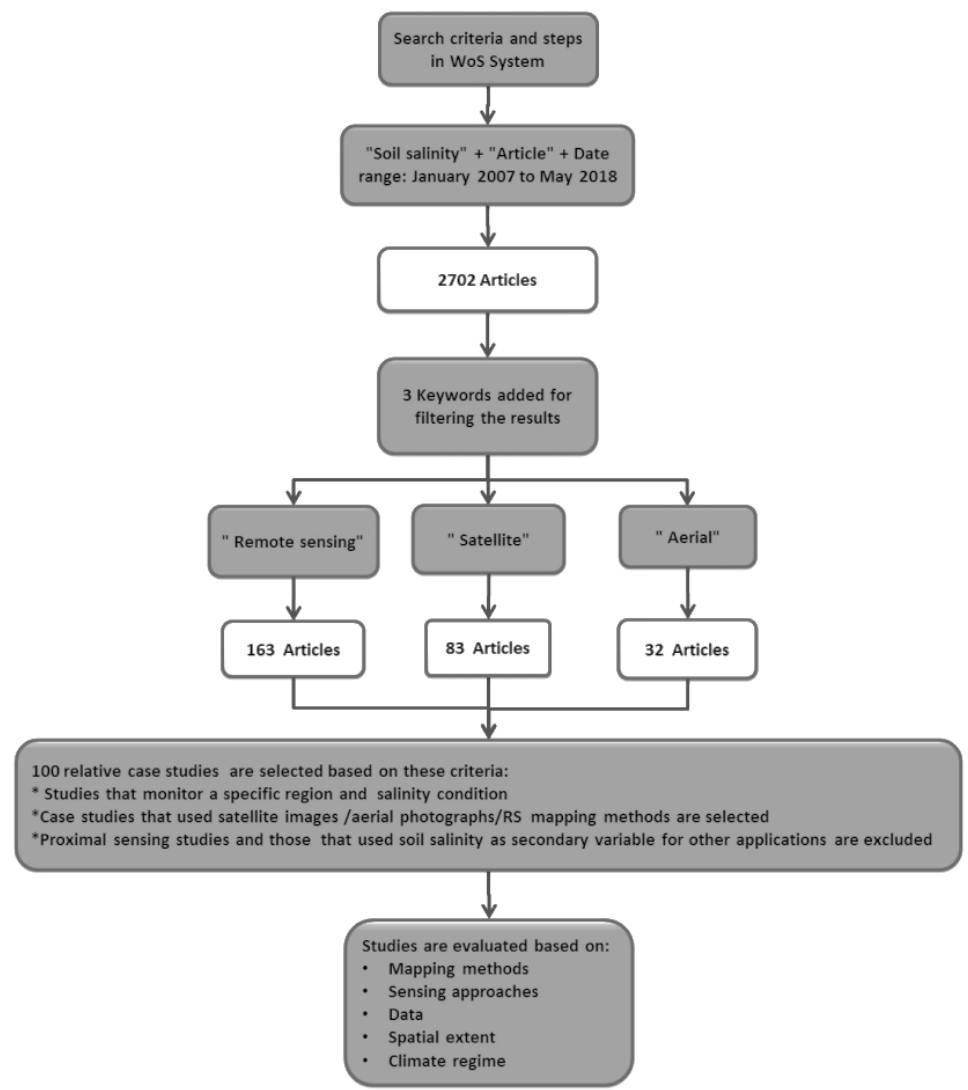

Figure 1. Flow diagram of the methodology used.

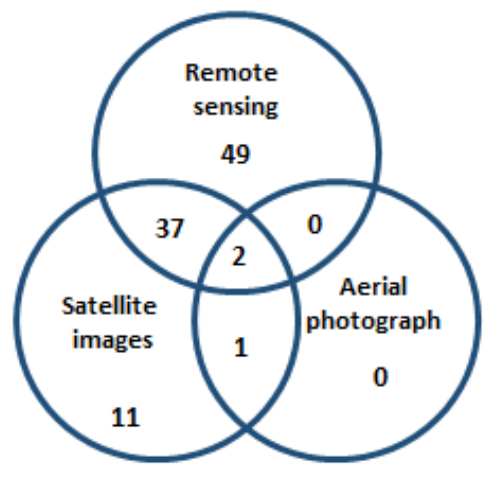

Figure 2. Number of selected articles based on keywords 


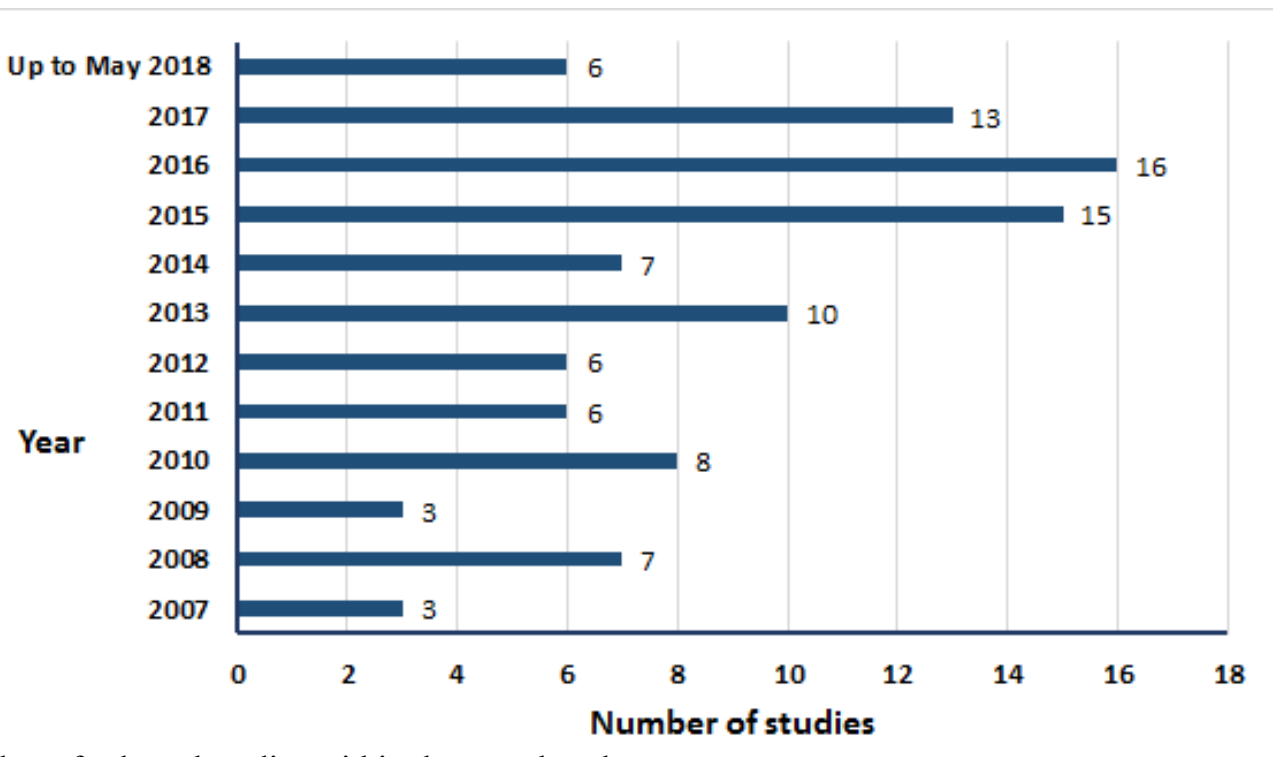

Figure 3. Number of selected studies within the past decade

Regarding to the geographical location and spatial extent of the selected case studies, this review reveals that soil salinity monitoring and detection has been surveyed locally and regionally in the 6 continents including more than 25 developing and developed countries. Majority of investigated studies were locally conducted; however, they were large areas underlining the utility of RS technology in monitoring vast areas. Therefore, experiencing soil salinization is becoming a more global concern and many nations are attempting to manage this environmental issue.

This study classifies sensing approaches of the selected studies based on 4 categories namely satellite images, aerial photography, field measurement and laboratory analysis. Except the two studies which utilized aerial photography as sensing approaches, all others applied satellite images, especially multispectral data. In addition, the matrix developed shows that field measurements carried out in most of the studies are usually conducted for correlating the real measured electrical conductivity (EC) values with estimated ones derived from RS mapping approaches. In some cases, laboratory analysis was also performed to provide more reliable data. In terms of data that is applied in each research, this review exposes that multispectral sensors including IKONOS, MODIS, IRS, Huan Jing (HJ)-1A, Quickbird, ASTER, Landsat series, WorldView2 (WV2) and SPOT have been used for exploring soil salinity studies with the aim of detecting, monitoring and mapping saline soils. It can be interpreted that Landsat series have been widely used in comparison to other multispectral data regarding the fact that this data is freely available in the global sense and spanning from 1972 till today. Considering the very high spatial resolution ( $1 \mathrm{~m}$ or better) of IKONOS, Quickbird and Worldview-2 and similar type of satellites; these RS data are mostly applicable to analyze small areas in local scale with high spatial details. Temporal resolution of MODIS satellite could provide more frequent observations to monitor soil salinity; however, having moderate spatial resolution $(100 \mathrm{~m}$ or coarser) is a drawback limiting the spatial details that could be obtained. On the other hand, this sensor is highly suitable to monitor large areas specifically at regional scale with repeated images. In addition, satellites with synthetic aperture radar (SAR) such as RADARSAT-1, 2, have been applied for some case studies. Moreover, Table 1 illustrates that EO-1 ALI is also used as hyperspectral imager for detecting soil salinization in some of the studies. 


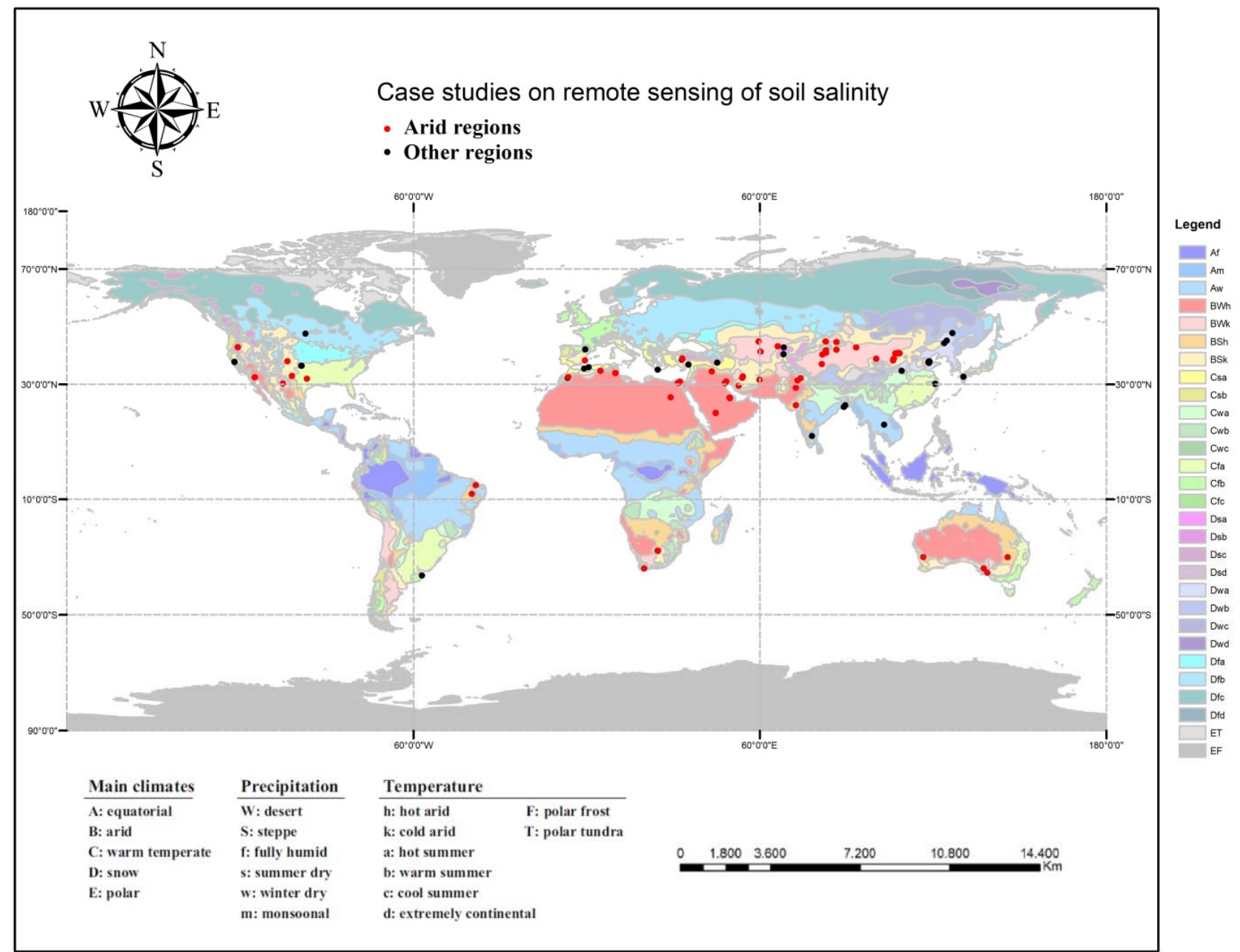

Figure 4. Geographical location of case studies selected for soil salinity monitoring via RS plotted on the KöppenGeiger Climate Classification map 
Table 1. Case studies on detecting and monitoring soil salinity via RS technology within 2007-2018

\begin{tabular}{|c|c|c|c|c|c|c|c|c|c|c|c|c|}
\hline \multirow{2}{*}{$\begin{array}{l}\text { Published } \\
\text { Year }\end{array}$} & \multirow{2}{*}{$\begin{array}{l}\text { Study } \\
\text { Year }\end{array}$} & \multirow{2}{*}{ Study Area } & \multirow{2}{*}{$\begin{array}{l}\text { Climate } \\
\text { Regime }\end{array}$} & \multirow{2}{*}{$\begin{array}{l}\text { Spatial } \\
\text { Extent }\end{array}$} & \multicolumn{4}{|c|}{ Sensing Approach } & \multirow{2}{*}{ Satellite Data } & \multirow[t]{2}{*}{ Analysis/ Mapping Methods } & \multirow{2}{*}{$\begin{array}{l}\text { Reason of } \\
\text { salinity }\end{array}$} & \multirow{2}{*}{ Reference } \\
\hline & & & & & SI* & AP* & $\mathbf{F M} *$ & $\mathbf{L A}^{*}$ & & & & \\
\hline 2018 & $2006-2008$ & $\begin{array}{c}\text { Junggar } \\
\text { Basin, China }\end{array}$ & BSk (Cold Arid Steppe) & local & $\cdot$ & & - & & MODIS & $\begin{array}{l}\text { Empirical model decomposition method, } \\
\text { linear and Random Forest }(\mathrm{RF}) \text { regression models }\end{array}$ & $\begin{array}{l}\text { Disturbance in soir }{ }^{2} \\
\text { water balance due to } \\
\text { environmental factors }\end{array}$ & $\begin{array}{l}\text { (Ma \& Yang, } \\
\text { 2018) }\end{array}$ \\
\hline 2018 & 2016 & $\begin{array}{l}\text { Ebinur Lake Wetland } \\
\text { National Nature } \\
\text { Reserve (ELWNNR), } \\
\text { Northwest China }\end{array}$ & BWk (Cold Arid Desert) & $\begin{array}{l}\text { local }(2670.8 \\
\left.\mathrm{km}^{2}\right)\end{array}$ & · & & . & - & $\begin{array}{l}\text { Landsat OLI, Huanjing } \\
\text { (HJ) 1-B CCD }\end{array}$ & $\begin{array}{l}\text { Develop optimal band Difference } \\
\text { Index (DI), Ratio Index (RII), and Normalization Index } \\
\text { (NDI) algorithms, } \\
\text { Bootstrap-BP neural network model }\end{array}$ & $\begin{array}{l}\text { Rare precipitation } \\
\text { and high evaporation }\end{array}$ & $\begin{array}{l}\text { (Wang et al., } \\
\text { 2018) }\end{array}$ \\
\hline 2018 & 2009-2011 & $\begin{array}{l}\text { Pompenillo, Grañén, } \\
\text { Spain }\end{array}$ & $\mathrm{Cfb}$ & local $\left(0.452 \mathrm{~km}^{2}\right)$ & $\cdot$ & & · & $\cdot$ & Landsat $5 \mathrm{TM}$ & Regression analysis and ordinary kriging (OK) & Parent material & (Casterad, 2018) \\
\hline 2018 & 2012 & $\begin{array}{l}\text { Zhangye Oasis, } \\
\text { Northwest China }\end{array}$ & BWk (Cold Arid Desert) & local & & • & $\cdot$ & & $\begin{array}{l}\text { Airborne hyperspectral } \\
\text { data }\end{array}$ & $\begin{array}{l}\text { Forced Invariance Approach, Generalized Linear Model } \\
\text { (GLM)-(generalization of ordinary linear regression) }\end{array}$ & & (Liu et al., 2018) \\
\hline 2018 & 2013 & $\begin{array}{l}\text { Inner Mongolia of } \\
\text { China }\end{array}$ & BWk (Cold Arid Desert) & local $\left(0.81 \mathrm{~km}^{2}\right)$ & . & & . & & Landsat 5 , Landsat 8 & $\begin{array}{l}\text { PLSR model, principal components analysis (PCA), } \\
\text { canonical correlation } \\
\text { analysis, linear regression analysis method }\end{array}$ & $\begin{array}{l}\text { Brackish water of flood } \\
\text { irrigation }\end{array}$ & (Zeng, 2018) \\
\hline 2018 & $\begin{array}{l}2012-2015- \\
2016\end{array}$ & Timpaki, Greece & Csa & local $\left(50 \mathrm{~km}^{2}\right)$ & . & & . & & WV2 images, Landsat 8 & $\begin{array}{c}\text { Regression analysis, } \\
\text { utilizing indices, } \\
\text { PCA }\end{array}$ & $\begin{array}{l}\text { Seawater intrusion due } \\
\text { to agricultural activities }\end{array}$ & $\begin{array}{l}\text { (Alexakis et al., } \\
\text { 2018) }\end{array}$ \\
\hline 2018 & 2016 & Bergama, Turkey & Csa & local & & & & & Sentinel-1A SAR & & & $\begin{array}{l}\text { (Şekertekin et al., } \\
\text { 2018) }\end{array}$ \\
\hline 2017 & 2006-2008 & Tarim Basin, China & BWk (Cold Arid Desert) & local & . & & . & & MODIS LST, ET & $\begin{array}{l}\text { Random Forest (RF) regression models, } \\
\text { Empirical mode decomposition (EMD) }\end{array}$ & $\begin{array}{l}\text { Meteorological and } \\
\text { geological factors, rising } \\
\text { groundwater } \\
\text { level }\end{array}$ & (Ma et al., 2017) \\
\hline 2017 & 2009 & South Khorasan, Iran & BWh (Hot Arid Desert) & local $\left(2487 \mathrm{~km}^{2}\right)$ & . & & . & & Landsat $\mathrm{ETM}^{+}$ & $\begin{array}{l}\text { Interpretation and } \\
\text { calculation of salinity index from satellite data } \\
\text { (Normalized Difference Salinity Index- NDSI) } \\
\text { Regression analysis }\end{array}$ & $\begin{array}{l}\text { Region retains water } \\
\text { and allows no outflow } \\
\text { due to its geologic } \\
\text { properties }\end{array}$ & ( Saghafi, 2017) \\
\hline 2017 & $2007-2013$ & $\begin{array}{l}\text { San Joaquin Valley, } \\
\text { California, USA }\end{array}$ & Csb & local $\left(17 \mathrm{~km}^{2}\right)$ & $\cdot$ & & · & $\cdot$ & Landsat 7 & $\begin{array}{l}\text { Utilizing Canopy Response Salinity Index } \\
\text { (CRSI), enhanced vegetation index and NDVI }\end{array}$ & $\begin{array}{c}\text { Drought, climate change, } \\
\text { reduced water } \\
\text { allocations, and land-use } \\
\text { changes }\end{array}$ & $\begin{array}{l}\text { (Scudiero et al., } \\
\text { 2017) }\end{array}$ \\
\hline 2017 & 2009-2016 & $\begin{array}{l}\text { Oran Watershed, } \\
\text { Algeria }\end{array}$ & Csa & local $\left(1878 \mathrm{~km}^{2}\right)$ & $\cdot$ & & · & & Landsat 8 OLI, TIRS & RBF interpolation, regression fitting model & Saline lake & (Abdellatif, 2017) \\
\hline 2017 & $\begin{array}{l}\text { 2001, 2005, } \\
\text { 2010, and } 2015\end{array}$ & Dharmapuri, India & Dfa & local $\left(430 \mathrm{~km}^{2}\right)$ & . & & . & & $\begin{array}{l}\text { Landsat 7-8 (ETM+ and } \\
\text { OLI), RADAR (SAR) }\end{array}$ & $\begin{array}{l}\text { Cross correlation analysis, simple linear regression } \\
\text { analysis }\end{array}$ & $\begin{array}{l}\text { Extreme temperature and } \\
\text { active parent fluoride } \\
\text { content in the } \\
\text { groundwater }\end{array}$ & $\begin{array}{l}\text { (Periasamy \& } \\
\text { Shanmugam, 2017) }\end{array}$ \\
\hline 2017 & 2005 & $\begin{array}{l}\text { Syrdarya Province of } \\
\text { Uzbekistan }\end{array}$ & BSk (Cold Arid Steppe) & local & · & & & & MODIS (Aqua Satellite) & $\begin{array}{l}\text { Utilizing enhanced vegetation index, temperature and } \\
\text { NDVI }\end{array}$ & & $\begin{array}{l}\text { (Ivushkin et al., } \\
\text { 2017) }\end{array}$ \\
\hline 2017 & 2013 & Gabes,Tunusia & BWh (Hot Arid Desert) & local $\left(300 \mathrm{~km}^{2}\right)$ & . & & . & . & $\begin{array}{l}\text { Landsat 8, ASTER- } \\
\text { GDEM2 }\end{array}$ & $\begin{array}{l}\text { Ordinary kriging (OK), minimum distance (MD) } \\
\text { classification, and simple regression (SR) }\end{array}$ & Parent material & $\begin{array}{l}\text { (Triki Fourati et } \\
\text { al., 2017) }\end{array}$ \\
\hline 2017 & 2010 & Xinjiang, China & BWk (Cold Arid Desert) & local & $\cdot$ & & . & $\cdot$ & Huan Jing (HJ)-1A & $\begin{array}{l}\text { Correlation analysis and partial least squares regression } \\
\text { (PLSR) model }\end{array}$ & $\begin{array}{l}\text { Parent material, rising } \\
\text { groundwater level }\end{array}$ & $\begin{array}{l}\text { (Jiang and } \mathrm{Xu} \text {, } \\
\text { 2018) }\end{array}$ \\
\hline 2017 & - & $\begin{array}{l}\text { Wadi ad-Dawasir, } \\
\text { Saudi Arabia }\end{array}$ & BWh (Hot Arid Desert) & local & $\cdot$ & & & & $\begin{array}{l}\text { Landsat } 8 \\
\text { OLI }\end{array}$ & $\begin{array}{l}\text { Principal component analysis (PCA), artificial } \\
\text { neural network (ANN) analyses }\end{array}$ & Poor drainage, irrigation & $\begin{array}{l}\text { (Elhag \& Bahrawi, } \\
\text { 2017) }\end{array}$ \\
\hline 2017 & $\begin{array}{l}\text { 1990, } 2002, \\
2000,2011 \text { and } \\
2015 \text {. }\end{array}$ & Turkey & BSk (Cold Arid Steppe) & local $\left(1500 \mathrm{~km}^{2}\right)$ & . & & . & & Landsat 5 TM, Landsat 8 & Utilizing salinity indices, linear regression analysis & Saline lake & $\begin{array}{l}\text { (Gorii et al., } \\
2017 \mathrm{a})\end{array}$ \\
\hline
\end{tabular}




\begin{tabular}{|c|c|c|c|c|c|c|c|c|c|c|}
\hline 2017 & 1984-2015 & $\begin{array}{l}\text { Biskra Area, } \\
\text { Algeria }\end{array}$ & BWh (Hot Arid Desert) & local $\left(5000 \mathrm{~km}^{2}\right)$ & . & & Landsat 5 , Landsat 8 & $\begin{array}{l}\text { Decision tree classification (DTC), } \\
\text { principle component analysis (PCA), utilizing spectral indices }\end{array}$ & Parent materials & $\begin{array}{l}\text { (Afrasinei et al., } \\
\text { 2017) }\end{array}$ \\
\hline 2017 & 2009 & Iran & Dsa & local $\left(50 \mathrm{~km}^{2}\right)$ & . & . & Landsat7 ETM+ + & PC analysis, MNF transformation, utilizing PPI, regression analysis & $\begin{array}{l}\text { Effects of endorheic basin } \\
\text { in retaining the water, } \\
\text { geologic structure }\end{array}$ & $\begin{array}{l}\text { (Rahmati and } \\
\text { Hamzehpour, } \\
\text { 2017) }\end{array}$ \\
\hline 2017 & & South Africa & BSh (Hot Arid Steppe) & $\begin{array}{c}\text { local }(0.3695 \\
\left.\mathrm{km}^{2}\right)\end{array}$ & & . & Aerial photography & Principal component analysis (PCA) Regression modeling, Kriging & $\begin{array}{l}\text { Insufficient natural } \\
\text { drainage }\end{array}$ & $\begin{array}{l}\text { (Vermeulen and } \\
\text { Niekerk, 2017) }\end{array}$ \\
\hline 2016 & $\begin{array}{l}1975-2000 \text { and } \\
2010\end{array}$ & $\begin{array}{l}\text { West Bengal, } \\
\text { India }\end{array}$ & Aw & local $\left(808 \mathrm{~km}^{2}\right)$ & - & . & Landsat MSS,TM and ETM+ & Kriging, utilizing indices (NDVI,SAVI,NDSI) & Seawater and wind impacts & $\begin{array}{l}\text { (Das et al, } \\
2016)\end{array}$ \\
\hline 2016 & 1985-2015 & $\begin{array}{l}\text { Yellow River } \\
\text { Delta, China }\end{array}$ & Dwa & local & · & & $\begin{array}{l}\text { Landsat-4/5 TM,Landsat-7 } \\
\text { ETM }+ \text {, Landsat-8 OLI }\end{array}$ & Partial Least Square Regression (PLSR model & $\begin{array}{l}\text { Seawater intrusion, } \\
\text { climate change effect }\end{array}$ & $\begin{array}{l}\text { (Fan et al., } \\
\text { 2016) }\end{array}$ \\
\hline 2016 & 2007-2008 & $\begin{array}{l}\text { Yellow River } \\
\text { Delta, China }\end{array}$ & Dwa & local & . & & Landsat TM & $\begin{array}{l}\text { The generalized additive model (GAM) with using spectral and terrain } \\
\text { indices } \\
\text { model - NDVI, RVI, DVI }\end{array}$ & $\begin{array}{l}\text { Seawater intrusion, } \\
\text { climate change effect }\end{array}$ & $\begin{array}{l}\text { (Chuangye et } \\
\text { al., 2016) }\end{array}$ \\
\hline 2016 & 2013 & $\begin{array}{l}\text { Yellow River } \\
\text { Delta, China }\end{array}$ & Dwa & local $\left(6650 \mathrm{~km}^{2}\right)$ & - & . & $\begin{array}{l}\text { Landsat-8( } \\
\text { OLI/TIRS), Landsat } 7 \text { ETM }+\end{array}$ & $\begin{array}{l}\text { Multi-linear regression model Utilizing satellite indices Combined } \\
\text { spectral response index (COSRI) , NDSI, BI }\end{array}$ & & $\begin{array}{l}\text { (Meng et al., } \\
\text { 2016) }\end{array}$ \\
\hline 2016 & 2000-2013 & Morocco & BSh (Hot Arid Steppe) & local & - & . & Landsat $5 \mathrm{TM}$, Landsat $8 \mathrm{OLI}$ & $\begin{array}{l}\text { Utilizing the Soil Salinity Spectral Indices (SSSI)-(OLI-SI index, } \\
\text { NDVI),simple linear } \\
\text { regression between sampled soil EC }\end{array}$ & $\begin{array}{l}\text { Poor irrigation and } \\
\text { agricultural practices, } \\
\text { climate change effect }\end{array}$ & (El et al., 2016) \\
\hline 2016 & 2012 & $\begin{array}{l}\text { Northern Cape, } \\
\text { South Africa }\end{array}$ & BSh (Hot Arid Steppe) & local & . & . & WorldView2 (WV2) sensor & $\begin{array}{l}\text { Principle component analysis (PCA), Regression analyses classification } \\
\text { and regression tree (CART) modelling }\end{array}$ & & $\begin{array}{l}\text { (Jacobus and } \\
\text { Niekerk, 2016a) }\end{array}$ \\
\hline 2016 & 2012-2013 & $\begin{array}{l}\text { Vaalharts and } \\
\text { Breede River, } \\
\text { South Africa }\end{array}$ & BSk (Cold Arid Steppe) & local & - & . & SPOT-5 & $\begin{array}{l}\text { Regression modelling (stepwise linear regression, partial least squares } \\
\text { regression, curve fit regression modelling) and supervised classification } \\
\text { (maximum likelihood, nearest neighbor, decision tree analysis, support } \\
\text { vector machine (SVM) and random forests) }\end{array}$ & & $\begin{array}{l}\text { (Jacobus and } \\
\text { Niekerk, } \\
\text { 2016b) }\end{array}$ \\
\hline 2016 & 2010-2011 & $\begin{array}{l}\text { Cukurova, } \\
\text { Turkey }\end{array}$ & Csa & local $\left(2170 \mathrm{~km}^{2}\right)$ & $\cdot$ & . & Landsat TM,ETM & $\begin{array}{l}\text { Object based classification, vegetation indices } \\
\text { (NDVI,NDWI,SAVI,GVI,PVI,WDVI,WETNESS), multi-linear } \\
\text { regression (MLR) and SLR, radial basis function technique }\end{array}$ & $\begin{array}{l}\text { Inappropriate irrigation } \\
\text { practices, high } \\
\text { groundwater table nearby } \\
\text { the sea }\end{array}$ & $\begin{array}{l}\text { (Satir and } \\
\text { Berberoglu, } \\
\text { 2016) }\end{array}$ \\
\hline 2016 & 2012 & South Africa & BSh (Hot Arid Steppe) & local $\left(100 \mathrm{~km}^{2}\right)$ & . & . & WorldView-2 image & Regression modeling and decision tree analysis (DCA) & $\begin{array}{l}\text { Insufficient natural } \\
\text { drainage }\end{array}$ & $\begin{array}{l}\text { (Vermeulen and } \\
\text { Niekerk, 2016) }\end{array}$ \\
\hline 2016 & 2002-2013 & $\begin{array}{l}\text { Western Desert, } \\
\text { Egypt }\end{array}$ & BWh (Hot Arid Desert) & local & . & $\cdot$ & Landsat 8 OLI & $\begin{array}{l}\text { Regression analysis, utilizing satellite image (SAVI } \\
\text { algorithm) }\end{array}$ & $\begin{array}{l}\text { High evapotranspiration } \\
\text { rates, Low precipitation }\end{array}$ & $\begin{array}{l}\text { (Hereher and } \\
\text { Ismael, 2016) }\end{array}$ \\
\hline 2016 & 2013-2015 & Northeast China & Dwb & local & $\cdot$ & $\cdot$ & Landsat 8 OLI & $\begin{array}{l}\text { Correlation analysis } \\
\text { regression model }\end{array}$ & & $\begin{array}{l}\text { (Bai et al., } \\
\text { 2016.) }\end{array}$ \\
\hline 2016 & 2012 and 2013 & Bangladesh & Aw & local & $\cdot$ & $\cdot$ & Landsat 7 ETM+ + & $\begin{array}{l}\text { Regression analysis, } \\
\text { utilizing salinity indices }\end{array}$ & Seawater intrusion & $\begin{array}{l}\text { (Morshed et al., } \\
\text { 2016) }\end{array}$ \\
\hline 2016 & 2007 & Morocco & Csa & local & . & . & EO-1 ALI & Utilizing salinity indices & $\begin{array}{l}\text { Excessive use of ground } \\
\text { water } \\
\text { High evapotranspiration } \\
\text { rates, Low precipitation }\end{array}$ & $\begin{array}{l}\text { (Bannari and } \\
\text { Guédon, 2016) }\end{array}$ \\
\hline 2016 & 2014 & Saudi Arabia & BWh (Hot Arid Desert) & local & . & . & Landsat 8 OLI & $\begin{array}{l}\text { Regression correlation } \\
\text { / Linear regression model }\end{array}$ & $\begin{array}{l}\text { High groundwater } \\
\text { abstraction rates }\end{array}$ & (Elhag,2016) \\
\hline 2016 & 2013 & $\begin{array}{l}\text { Yellow River } \\
\text { Delta, China }\end{array}$ & Dwa & local & - & - & Landsat 7- Landsat 8 & Multiple linear regression, kriging & $\begin{array}{l}\text { High evapotranspiration } \\
\text { rates, low precipitation }\end{array}$ & $\begin{array}{l}\text { (An et al., } \\
\text { 2016) }\end{array}$ \\
\hline 2016 & 1987 to 2014 & $\begin{array}{c}\text { South } \\
\text { Kazakhstan }\end{array}$ & DSa & local & . & & Pleiades 1A / 1B, LANDSAT TM & Regression analysis & & $\begin{array}{l}\text { (Laiskhanov et } \\
\text { al., 2016) }\end{array}$ \\
\hline 2015 & 2013 & Gabes, Tunisia & BWh (Hot Arid Desert) & local & - & . & Landsat-8 & $\begin{array}{l}\text { Partial least square regression (PLSR) method; utilizing spectral } \\
\text { salinity indices }\end{array}$ & Parent material & $\begin{array}{l}\text { (Triki Fourati et } \\
\text { al., 2015) }\end{array}$ \\
\hline 2015 & $2012-2013$ & Algeria & Csa & local & . & . & Landsat $\mathrm{ETM}^{+}$ & Multiple linear regression model & & $\begin{array}{l}\text { (Yahiaoui et al., } \\
\text { 2015) }\end{array}$ \\
\hline 2015 & 2007-2013 & $\begin{array}{l}\text { Western San } \\
\text { Joaquin Valley, } \\
\text { USA }\end{array}$ & Csb & local & . & . & Landsat 7 & Utilizing canopy response salinity index (CRSI) & $\begin{array}{l}\text { Extensive agricultural } \\
\text { practices }\end{array}$ & $\begin{array}{l}\text { (Scudiero et } \\
\text { al., 2015) }\end{array}$ \\
\hline 2015 & 2014 & $\begin{array}{l}\text { East Nile Delta } \\
\text { Region, Egypt }\end{array}$ & BWh (Hot Arid Desert) & local & - & - & Landsat 8, ASTER GDEM & $\begin{array}{l}\text { Principal component analysis, } \\
\text { utilizing salinity indices }\end{array}$ & $\begin{array}{l}\text { Poor quality groundwater, } \\
\text { inappropriate irrigation and } \\
\text { anthropogenic activities }\end{array}$ & $\begin{array}{l}\text { (Arnous et al., } \\
2015)\end{array}$ \\
\hline
\end{tabular}


Gorji, et al. / IJEGEO (6)1: 33-49 (2019)

\begin{tabular}{|c|c|c|c|c|c|c|c|c|c|c|c|}
\hline 2015 & 2013 & Brazil & BSh (Hot Arid Steppe) & local & . & . & . & OL/Landsat-8,Hyperion & $\begin{array}{l}\text { Principal component analysis (PCA), } \\
\text { support vector machine (SVM), } \\
\text { regression analysis }\end{array}$ & & $\begin{array}{l}\text { (Moreira et al., } \\
\text { 2015) }\end{array}$ \\
\hline 2015 & $\begin{array}{l}2008,2011- \\
2013\end{array}$ & $\begin{array}{l}\text { Keriya River } \\
\text { Basin, } \\
\text { Northwestern } \\
\text { China }\end{array}$ & BWk (Cold Arid Desert) & local & . & . & & $\begin{array}{l}\text { Landsat ETM+, PALSAR and } \\
\text { Radarsat-2 }\end{array}$ & $\begin{array}{l}\text { Support vector machine (SVM) classification, decision tree (DT) } \\
\text { classifier }\end{array}$ & $\begin{array}{l}\text { High evapotranspiration, } \\
\text { low precipitation }\end{array}$ & $\begin{array}{l}\text { (Nurmemet et } \\
\text { al., 2015) }\end{array}$ \\
\hline 2015 & 2009 & Xinjiang, China & BWk (Cold Arid Desert) & local & . & . & . & Hyperion & $\begin{array}{l}\text { Maximum likelihood, k-nearest neighbor, support vector machine } \\
\text { (SVM), neural network, and the } \\
\text { spectral feature parameters algorithms }\end{array}$ & & (Jin et al., 2015) \\
\hline 2015 & $\begin{array}{l}1984,2000 \\
2006 \text { and } 2013\end{array}$ & $\begin{array}{l}\text { Nile Delta } \\
\text { Region, Egypt }\end{array}$ & BWh (Hot Arid Desert) & local & . & . & & Landsat $5,7,8$-ASTER & $\begin{array}{l}\text { Supervised and unsupervised classification (maximum likelihood } \\
\text { classification), Principal Components Analysis (PCA), and indices }\end{array}$ & - & $\begin{array}{l}\text { (Arnous and } \\
\text { Green, 2015) }\end{array}$ \\
\hline 2015 & - & $\begin{array}{l}\text { Western } \\
\text { Australia }\end{array}$ & BSh (Hot Arid Steppe) & local & . & & & Landsat TM & $\begin{array}{l}\text { Linear mixed effect model, } \\
\text { utilizing NDVI index }\end{array}$ & & $\begin{array}{l}\text { (Kobryn et al., } \\
\text { 2015) }\end{array}$ \\
\hline 2015 & 2014 & $\begin{array}{l}\text { West Texas } \\
\text { (Frost and Silver } \\
\text { Lake). USA }\end{array}$ & Cfa-BSk & local & . & . & . & Landsat 8 & Regression and correlation analysis & Saline playas & $\begin{array}{l}\text { (Aldabaa et al., } \\
2015 \text { ) }\end{array}$ \\
\hline 2015 & & $\begin{array}{l}\text { Yellow River } \\
\text { Delta, China }\end{array}$ & Dwa & local & . & . & . & $\begin{array}{l}\text { Landsat } 7 \text { ETM+, EO-1 ALI and } \\
\text { Landsat } 8 \text { OLI }\end{array}$ & Partial least square regression (PLSR) model & & $\begin{array}{l}\text { (Fan et al., } \\
\text { 2015) }\end{array}$ \\
\hline 2015 & 2013 & Gujarat, India & BSh (Hot Arid Steppe) & local & . & . & & Hyperion & $\begin{array}{l}\text { Utilizing spectral angle mapper (SAM) method, regression analysis } \\
\text { (PLS) method }\end{array}$ & & $\begin{array}{l}\text { (Mitran et al., } \\
\text { 2015) }\end{array}$ \\
\hline 2015 & 2008 & $\begin{array}{l}\text { Yellow River } \\
\text { Delta, China }\end{array}$ & Dwa & local & . & . & & MODIS & Correlation and regression analysis & & $\begin{array}{l}\text { (Zhang et al., } \\
\text { 2015) }\end{array}$ \\
\hline 2015 & 1998-1999 & Southern Japan & $\mathrm{Cfa}$ & local & . & $\cdot$ & & Landsat 5 & Utilizing NDVI index & Tsunamis and typhoons & $\begin{array}{l}\text { (Goto et al., } \\
\text { 2014) }\end{array}$ \\
\hline 2015 & 2013 & $\begin{array}{l}\text { Buenos Aires, } \\
\text { Argentina }\end{array}$ & $\mathrm{Cfb}$ & local & . & . & & Quick Bird & Correlation analysis & & $\begin{array}{l}\text { (Carol et al., } \\
\text { 2015) }\end{array}$ \\
\hline 2014 & 2011 & $\begin{array}{l}\text { Werigan-Kuqa } \\
\text { Oasis, China }\end{array}$ & BWk (Cold Arid Desert) & local & . & . & . & Landsat-TM & $\begin{array}{c}\text { Regression kriging interpolation, spectral index regression, universal } \\
\text { kriging }\end{array}$ & $\begin{array}{l}\text { Extreme continental } \\
\text { climatic condition and } \\
\text { irrational irrigation } \\
\text { activities }\end{array}$ & $\begin{array}{l}\text { (Ding and } Y u \text {, } \\
\text { 2014) }\end{array}$ \\
\hline 2014 & 2009-2012 & $\begin{array}{l}\text { Mesopotamia, } \\
\text { Iraq }\end{array}$ & BWh (Hot Arid Desert) & local & . & . & & Landsat ETM+ and MODIS & $\begin{array}{l}\text { Multiple linear } \\
\text { regression analysis }\end{array}$ & $\begin{array}{l}\text { Water-table rising and } \\
\text { water-logging due to } \\
\text { irrigation }\end{array}$ & $\begin{array}{l}\text { (Wu et al., } \\
\text { 2014) }\end{array}$ \\
\hline 2014 & 2006 and 2012 & $\begin{array}{l}\text { El-Tina Plain, } \\
\text { Sinai, Egypt }\end{array}$ & BWh (Hot Arid Desert) & local & . & . & & $\begin{array}{l}\text { Landsat } 7 \text { ETM+ }+ \text {, Landsat } 5 \\
\text { Thematic Mapper (TM) }\end{array}$ & PLSR and MARS & $\begin{array}{l}\text { Subsurface seepage of } \\
\text { seawater, high salinity of } \\
\text { the groundwater table, } \\
\text { irrational irrigation }\end{array}$ & $\begin{array}{l}\text { (Nawar et al., } \\
2014)\end{array}$ \\
\hline 2014 & 2012 & $\begin{array}{l}\text { Al-Hassa Oasis, } \\
\text { Saudi Arabia }\end{array}$ & BWh (Hot Arid Desert) & local & . & . & & IKONOS & Utilizing salinity indices & $\begin{array}{l}\text { Topography, poor } \\
\text { drainage, poor irrigation } \\
\text { and water quality, } \\
\text { mismanaged agricultural } \\
\text { practices }\end{array}$ & $\begin{array}{l}\text { (Allbed et al., } \\
\text { 2014a) }\end{array}$ \\
\hline 2014 & 2012 & $\begin{array}{l}\text { Al Hassa Oasis, } \\
\text { Saudi Arabia }\end{array}$ & BWh (Hot Arid Desert) & local & . & . & & IKONOS & Regression models & High p evaporation rate & $\begin{array}{l}\text { (Allbed et al., } \\
\text { 2014b) }\end{array}$ \\
\hline 2014 & 2009 & $\begin{array}{l}\text { Pingluo County, } \\
\text { China }\end{array}$ & BWk (Cold Arid Desert) & local $\left(34 \mathrm{~km}^{2}\right)$ & . & . & & QuickBird & $\begin{array}{l}\text { Utilizing spectral indices, statistical analysis, partial least squares } \\
\text { regression analysis (PLSR) method }\end{array}$ & $\begin{array}{l}\text { High groundwater table, } \\
\text { high evaporation rate and } \\
\text { irrational } \\
\text { irrigation }\end{array}$ & $\begin{array}{l}\text { (Sidike et al., } \\
\text { 2014) }\end{array}$ \\
\hline 2014 & 2011 & $\begin{array}{c}\text { Maha- } \\
\text { Sarahkam } \\
\text { Province,Thaila } \\
\text { nd }\end{array}$ & $\mathrm{Aw}$ & local $\left(400 \mathrm{~km}^{2}\right)$ & 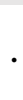 & . & & ALOS-PALSAR & Neural network model & & $\begin{array}{l}\text { (Phonphan et } \\
\text { al., 2014) }\end{array}$ \\
\hline 2013 & 1998-2008 & Europe & - & continental & . & & & SPOT & $\begin{array}{l}\text { Utilizing NDVI, } \\
\text { moving average (MA) algorithm }\end{array}$ & & $\begin{array}{l}\text { (Ivits et al., } \\
2013 \text { ) }\end{array}$ \\
\hline 2013 & 2003 & $\begin{array}{l}\text { Shangyu City } \\
\text {,China }\end{array}$ & $\mathrm{Cfa}$ & $\begin{array}{l}\text { local }(260.61 \\
\left.\mathrm{km}^{2}\right)\end{array}$ & . & . & . & QuickBird & Fuzzy k-means (FKM) algorithm & & $\begin{array}{l}\text { ( Guo et al., } \\
2013 \text { ) }\end{array}$ \\
\hline 2013 & 2007 & $\begin{array}{l}\text { Taklimakan } \\
\text { Desert, China }\end{array}$ & BWk (Cold Arid Desert) & local $\left(2500 \mathrm{~km}^{2}\right)$ & . & . & - & Landsat $5 \mathrm{TM}$ & $\begin{array}{l}\text { Utilizing salinity indices, } \\
\text { Exponential regression analysis }\end{array}$ & & $\begin{array}{l}\text { (Wang et al., } \\
\text { 2013) }\end{array}$ \\
\hline 2013 & 2009 & $\begin{array}{l}\text { Guanzhong } \\
\text { Plain, Northern } \\
\text { China }\end{array}$ & Dwa & local $\left(0.64 \mathrm{~km}^{2}\right)$ & . & . & & MODIS & Ordinary kriging method & $\begin{array}{l}\text { Mineral material in } \\
\text { groundwater }\end{array}$ & $\begin{array}{l}\text { (Quan et al., } \\
\text { 2013) }\end{array}$ \\
\hline 2013 & 1999 & $\begin{array}{l}\text { Fars Province, } \\
\text { Iran }\end{array}$ & BSk (Cold Arid Steppe) & local $\left(950 \mathrm{~km}^{2}\right)$ & & $\cdot$ & & MODIS & $\begin{array}{l}\text { Principal component analysis (PCA), simple correlation techniques, } \\
\text { stepwise multiple linear regression techique, kriging method }\end{array}$ & Saline and alkaline water & $\begin{array}{l}\text { (Fallah Shamsi } \\
\text { et al., 2013) }\end{array}$ \\
\hline 2013 & 1992-1995 & Punjab, Pakistan & BWh (Hot Arid Desert) & local & & & & IRS-1B LISS-II & Supervised classification, using & & (Abbas et al., \\
\hline
\end{tabular}


Gorji, et al. / IJEGEO (6)1: 33-49 (2019)

\begin{tabular}{|c|c|c|c|c|c|c|c|c|c|c|c|}
\hline 2013 & 2010 & $\begin{array}{l}\text { Shangyu City, } \\
\text { Zhejiang } \\
\text { Province, China }\end{array}$ & $\mathrm{Cfa}$ & $\begin{array}{l}\text { local }(260.61 \\
\left.\mathrm{km}^{2}\right)\end{array}$ & . & & - & ALOS/PALSAR & $\begin{array}{l}\text { maximum likelihood algorithm } \\
\text { Inversion model based on a backscartering coefficient } \\
\text { semi-variogram, } \\
\text { kriging and co-kriging }\end{array}$ & Parent material & $\begin{array}{l}\text { 2013) } \\
\text { (Guo et al., } \\
\text { 2013) }\end{array}$ \\
\hline 2013 & 2008 & South Australia & BSk (Cold Arid Steppe) & local $\left(159 \mathrm{~km}^{2}\right)$ & $\cdot$ & & . & QuickBird & Unsupervised classification and utilizing salinity indices & $\begin{array}{l}\text { Patches of dry saline land } \\
\text { and dryland salinity }\end{array}$ & $\begin{array}{l}\text { (Setia et al., } \\
\text { 2013) }\end{array}$ \\
\hline 2013 & 2010 & Khuzestan, Iran & BWh (Hot Arid Desert) & local $\left(774 \mathrm{~km}^{2}\right)$ & . & & . & Hyperion & Linear regression analysis & $\begin{array}{l}\text { Presence of salt-containing } \\
\text { layers, parent material, } \\
\text { irrigation with saline } \\
\text { water, } \\
\text { high groundwater table, } \\
\text { high evaporation rates, } \\
\text { sediment transport during } \\
\text { flood periods and seawater } \\
\text { intrusion }\end{array}$ & $\begin{array}{l}\text { (Hamzeh et al., } \\
\text { 2013) }\end{array}$ \\
\hline 2013 & $\begin{array}{l}1975 \text { and } 2004 \\
2005\end{array}$ & $\begin{array}{l}\text { Ardakan Area } \\
\text {,Iran }\end{array}$ & BSk (Cold Arid Steppe) & local & $\cdot$ & & . & Landsat MSS and TM & Supervised classification & & $\begin{array}{l}\text { (Matinfar et al., } \\
\text { 2013) }\end{array}$ \\
\hline 2012 & 1990-2003 & $\begin{array}{l}\text { Basrah } \\
\text { Province, Iraq }\end{array}$ & BWh (Hot Arid Desert) & $\begin{array}{l}\text { local }(19070 \\
\left.\mathrm{km}^{2}\right)\end{array}$ & - & & - & . $\quad$ Landsat 5 TM, Landsat 7 ETM+ + & $\begin{array}{c}\text { Digital image classification and } \\
\text { band match methods. } \\
\text { land use } \\
\text { classification using both unsupervised and supervised } \\
\text { techniques }\end{array}$ & $\begin{array}{l}\text { High evapotranspiration } \\
\text { rate, low precipitation, } \\
\text { high mineralization } \\
\text { of groundwater, human- } \\
\text { induced activities }\end{array}$ & $\begin{array}{c}\text { (Jabbar \& } \\
\text { Zhou, 2012) }\end{array}$ \\
\hline 2012 & 2007 & Mexico & BWh (Hot Arid Desert) & local $\left(920 \mathrm{~km}^{2}\right)$ & . & & . & $\begin{array}{l}\text { Landsat } 5 \text { Thematic Mapper } \\
\text { (TM) }\end{array}$ & Regression analysis, utilizing indices & & $\begin{array}{l}\text { (Judkins \& } \\
\text { Myint, 2012) }\end{array}$ \\
\hline 2012 & 2005 & $\begin{array}{l}\text { Yinchuan Plain } \\
\text {,China }\end{array}$ & BWk (Cold Arid Desert) & local $\left(7790 \mathrm{~km}^{2}\right)$ & . & & - & MODIS & $\begin{array}{l}\text { Utilizing NDVI index, } \\
\text { correlation analysis }\end{array}$ & & (Jin et al., 2012) \\
\hline 2012 & 2006 to 2009 & $\begin{array}{l}\text { Jezre'el Valley, } \\
\text { Israel }\end{array}$ & Csa & local $\left(0.8 \mathrm{~km}^{2}\right)$ & & . & & AISA-Dual sensor & Partial least squares regression model, correlation analysis & & $\begin{array}{l}\text { (Goldshleger et } \\
\text { al.,2012) }\end{array}$ \\
\hline 2012 & 2002 & $\begin{array}{l}\text { Aral Sea Basin, } \\
\text { Uzbekistan }\end{array}$ & BWk (Cold Arid Desert) & local $\left(15 \mathrm{~km}^{2}\right)$ & - & & - & Landsat 7 & Neural network model & & $\begin{array}{l}\text { (Akramkhanov } \\
\text { \& Vlek, 2012) }\end{array}$ \\
\hline 2012 & $\begin{array}{l}1985,1999, \text { and } \\
2006\end{array}$ & $\begin{array}{l}\text { Yellow River } \\
\text { Delta, China }\end{array}$ & Dwa & local & - & & - & Landsat TM & $\begin{array}{l}\text { Utilizing NDVI and salinity indices, } \\
\text { Regression analysis }\end{array}$ & & $\begin{array}{l}\text { (Fan et al., } \\
\text { 2012) }\end{array}$ \\
\hline 2011 & 2009 & $\begin{array}{l}\text { Yinchuan Plain, } \\
\text { China }\end{array}$ & BWk (Cold Arid Desert) & local $\left(7793 \mathrm{~km}^{2}\right)$ & . & & - & HJ - I & $\begin{array}{l}\text { Support vector machine (SVM) } \\
\text { classification }\end{array}$ & $\begin{array}{l}\text { Topography, hydrology, } \\
\text { irrational agricultural } \\
\text { practices }\end{array}$ & $\begin{array}{l}\text { (Meimei \& } \\
\text { Ping, 2011) }\end{array}$ \\
\hline 2011 & 2008 & Northeast Brazil & $\mathrm{Aw}, \mathrm{BSh}, \mathrm{BWh}$ & regional & . & & . & MODIS & $\begin{array}{l}\text { Linear spectral unmixing technique (LSU), } \\
\text { utilizing salinity indices, } \\
\text { multiple linear regression }\end{array}$ & $\begin{array}{l}\text { Inappropriate irrigation, } \\
\text { parent material }\end{array}$ & $\begin{array}{l}\text { (Bouaziz et al., } \\
\text { 2011) }\end{array}$ \\
\hline 2011 & 2001 & $\begin{array}{l}\text { Central Punjab } \\
\text { Province, } \\
\text { Pakistan }\end{array}$ & BSh (Hot Arid Steppe) & local & . & & . & Landsat 7 ETM+ + & Utilizing salinity indices & High evaporation & (Iqbal, 2011) \\
\hline 2011 & 1990-2002 & $\begin{array}{l}\text { Khorezm } \\
\text { Province, } \\
\text { Uzbekistan }\end{array}$ & BWk (Cold Arid Desert) & local & . & & . & Landsat 7 & $\begin{array}{l}\text { Utilizizing spectral indices of normalized difference vegetation indices } \\
\text { (TNDVI), soil-adjusted } \\
\text { vegetation index (SAVI) and ratio vegetation index (RVI), } \\
\text { regression analysis }\end{array}$ & Shallow groundwater table & $\begin{array}{l}\text { (Akramkhanov } \\
\text { et al., 2011) }\end{array}$ \\
\hline 2011 & 1995 & $\begin{array}{l}\text { Indira Gandhi } \\
\text { Mukhya } \\
\text { Nahar, India }\end{array}$ & BWh (Hot Arid Desert) & local & . & & . & IRS LISS II & $\begin{array}{l}\text { Utilizing NDVI and SAVI indices } \\
\text { visual interpretation }\end{array}$ & $\begin{array}{l}\text { Flood irrigation practice, } \\
\text { canal } \\
\text { seepage, sandy soil texture } \\
\text { and absence of natural } \\
\text { surface drainage }\end{array}$ & $\begin{array}{l}\text { (Mandal \& } \\
\text { Sharma, 2011) }\end{array}$ \\
\hline 2011 & 2000 & $\begin{array}{l}\text { Al-Hassa Oasis, } \\
\text { Saudi Arabia }\end{array}$ & BWh (Hot Arid Desert) & local & - & & $\cdot$ & Landsat TM 5 & Utilizing NDVI and SAVI indices & Irrigation water & $\begin{array}{l}\text { (Aldakheel, } \\
\text { 2011) }\end{array}$ \\
\hline 2010 & 1991-2005 & $\begin{array}{l}\text { Inner Mongolia, } \\
\text { China }\end{array}$ & BWk (Cold Arid Desert) & local & . & & . & Landsat TM, ETM+ & $\begin{array}{l}\text { Supervised classification and visual interpretation, } \\
\text { maximum-likelihood supervised method }\end{array}$ & $\begin{array}{l}\text { Unfavorable topography, } \\
\text { geomorphology, soil } \\
\text { texture and irrigation??? }\end{array}$ & $\begin{array}{l}\text { (Yu et al., } \\
2010)\end{array}$ \\
\hline 2010 & 1986 to 2005 & $\begin{array}{l}\text { Rio Conchos } \\
\text { Basin in } \\
\text { Northern } \\
\text { Mexico }\end{array}$ & BSk (Cold Arid Steppe) & local & . & & . & Landsat 5 & $\begin{array}{l}\text { Principal Component analysis (PCA), } \\
\text { maximum likelihood classification, } \\
\text { visual interpretation }\end{array}$ & $\begin{array}{l}\text { Mineral deposits scattered } \\
\text { throughout the area }\end{array}$ & $\begin{array}{l}\text { (Gutierrez and } \\
\text { Johnson, 2010) }\end{array}$ \\
\hline 2010 & $\begin{array}{l}2000,2004 \text { and } \\
\quad 2007\end{array}$ & $\begin{array}{l}\text { Southern } \\
\text { Alicante } \\
\text { Province, Spain }\end{array}$ & BSk (Cold Arid Steppe) & local & . & & . & ASTER & $\begin{array}{l}\text { Matched filtering (MF) and mixture tuned matched filtering (MTMF), } \\
\text { linear spectral unmixing (LSU) }\end{array}$ & Inappropriate Irrigation & $\begin{array}{l}\text { (Melendez- } \\
\text { Pastor et al., } \\
2010 \text { ) }\end{array}$ \\
\hline 2010 & 1983-2000 & $\begin{array}{l}\text { Golodnaya } \\
\text { Steppe, } \\
\text { Uzbekistan }\end{array}$ & Dsa & local $\left(8 \mathrm{~km}^{2}\right)$ & $\cdot$ & . & . & Landsat 7,aerial photo, & Visual interpretation & $\begin{array}{l}\text { Irrigation water include } \\
\text { high content of salts with a } \\
\text { predominant } \\
\text { of chlorides and sulfates }\end{array}$ & $\begin{array}{l}\text { (Rukhovich et } \\
\text { al., 2010) }\end{array}$ \\
\hline 2010 & $2000-2006$ & $\begin{array}{l}\text { Southern } \\
\text { Colorado, USA }\end{array}$ & BSk (Cold Arid Steppe) & local & - & & - & $\begin{array}{l}\text { Landsat } 5 \text {, } \\
\text { Landsat } 7\end{array}$ & $\begin{array}{l}\text { Regression analysis } \\
\text { ordinary kriging, regression kriging, and co-kriging }\end{array}$ & $\begin{array}{l}\text { Salinity levels in the } \\
\text { irrigation canals }\end{array}$ & $\begin{array}{l}\text { (Lobell et al., } \\
2010)\end{array}$ \\
\hline
\end{tabular}




\begin{tabular}{|c|c|c|c|c|c|c|c|c|c|c|c|}
\hline 2010 & 2006 & $\begin{array}{l}\text { Southeastern } \\
\text { Oregon, } \\
\text { USA }\end{array}$ & BSk (Cold Arid Steppe) & local & . & . & & Landsat TM & $\begin{array}{l}\text { Decision-tree analysis (DTA), } \\
\text { utilizing salinity indices }\end{array}$ & $\begin{array}{l}\text { Irrigation water flows to } \\
\text { nearby low-lying areas } \\
\text { resulting in higher } \\
\text { groundwater table }\end{array}$ & $\begin{array}{l}\text { (Elnaggar \& } \\
\text { Noller, 2009) }\end{array}$ \\
\hline 2010 & 2005 & $\begin{array}{l}\text { Yellow River } \\
\text { Delta,China }\end{array}$ & Dwa & local & . & . & & EO-1 Hyperion & $\begin{array}{l}\text { Utilizing soil salinity spectral index } \\
\text { (SSI) and applying univariate regression analysis }\end{array}$ & $\begin{array}{l}\text { Low and flat terrain, high } \\
\text { groundwater table, high } \\
\text { mineralization rate, poor } \\
\text { drainage conditions, } \\
\text { infiltration and seawater } \\
\text { intrusion }\end{array}$ & $\begin{array}{l}\text { (WENG et al., } \\
\text { 2010) }\end{array}$ \\
\hline 2010 & 2006 & $\begin{array}{l}\text { South Dakota, } \\
\text { USA }\end{array}$ & Dfb & $\begin{array}{l}\text { local }(17,000 \\
\left.\mathrm{km}^{2}\right)\end{array}$ & - & . & & MODIS & Correlation and regression analyses & & $\begin{array}{l}\text { (Lobell et al., } \\
2010)\end{array}$ \\
\hline 2009 & 2007 & $\begin{array}{l}\text { Hetao Irrigation } \\
\text { District, Inner } \\
\text { Mongolia }\end{array}$ & BWk (Cold Arid Desert) & local & . & . & & Landsat TM & Partial least squares regression model & & $\begin{array}{l}\text { (Yong-hua et } \\
\text { al.,2009) }\end{array}$ \\
\hline 2009 & 2005 & Fengqui, China & Dwa & local & . & . & & Landsat TM & Regression models & & $\begin{array}{l}\text { (Ya-kun et al., } \\
\text { 2009) }\end{array}$ \\
\hline 2009 & 2003-2004 & $\begin{array}{l}\text { Point Sturt } \\
\text { Peninsula, } \\
\text { Australia }\end{array}$ & BSk (Cold Arid Steppe) & local $\left(140 \mathrm{~km}^{2}\right)$ & . & . & . & Hyperion & $\begin{array}{l}\text { Partial spectral unmixing techniques, } \\
\text { matched filtering (MF), } \\
\text { and mixture tuned matched filtering (MTMF) }\end{array}$ & $\begin{array}{l}\text { Quaternary and Tertiary } \\
\text { sediments that contain vast } \\
\text { salt stores }\end{array}$ & $\begin{array}{l}\text { (Dutkiewicz et } \\
\text { al., 2009) }\end{array}$ \\
\hline 2008 & 2004 & Colorado, USA & $\mathrm{Cfa}$ & Local $\left(1056 \mathrm{~km}^{2}\right)$ & . & $\cdot$ & & $\begin{array}{l}\text { Ikonos, } \\
\text { Landsat } 5\end{array}$ & $\begin{array}{l}\text { Ordinary least squares model, } \\
\text { ordinary kriging }\end{array}$ & - & $\begin{array}{l}\text { (Eldeiry et al., } \\
\text { 2008) }\end{array}$ \\
\hline 2008 & $1973-2006$ & $\begin{array}{l}\text { Inner Mongolia, } \\
\text { China }\end{array}$ & Bsk (Cold Arid Steppe) & local & . & $\cdot$ & & Landsat MSS, TM & Maximum likelihood classification & $\begin{array}{l}\text { Poor quality Irrigation } \\
\text { water }\end{array}$ & $\begin{array}{l}\text { (Wu et al., } \\
2008)\end{array}$ \\
\hline 2008 & 2006 & $\begin{array}{l}\text { Salt Lake, } \\
\text { Turkey }\end{array}$ & BSk (Cold Arid Steppe) & local- $\left(1500 \mathrm{~km}^{2}\right)$ & . & . & & Landsat- $5 \mathrm{TM}$ & Regression analysis & & $\begin{array}{c}\text { (Ekercin \& } \\
\text { Ormeci,2008) }\end{array}$ \\
\hline 2008 & 2000-2002 & $\begin{array}{l}\text { New South } \\
\text { Wales, Australia }\end{array}$ & BSh (Hot Arid Steppe) & local- $\left(900 \mathrm{~km}^{2}\right)$ & . & . & . & Landsat- $5 \mathrm{TM}$ & $\begin{array}{l}\text { Utilizing salinity index (SI), normalized difference salinity index } \\
\text { (NDSI) and brightness index (BI), supervised fuzzy classification, } \\
\text { regression-kriging }\end{array}$ & - & $\begin{array}{l}\text { (Odeh \& Onus, } \\
\text { 2008) }\end{array}$ \\
\hline 2008 & 2004 & $\begin{array}{l}\text { Tadla Region, } \\
\text { Morocco }\end{array}$ & Csa & local- $3600 \mathrm{~km}^{2}$ ) & . & - & - & $\begin{array}{l}\text { Advanced Land } \\
\text { Imaging (EO-1) }\end{array}$ & Utilizing salinity indices & - & $\begin{array}{l}\text { (Bannari et al., } \\
\text { 2008) }\end{array}$ \\
\hline 2008 & 1996 & $\begin{array}{c}\text { Ardakan, } \\
\text { Damghan, Lut } \\
\text { Desert (Yardang } \\
\text { Area), } \\
\text { Abarkooh, Qom, } \\
\text { Iran }\end{array}$ & BSk (Cold Arid Steppe) & regional & . & . & & Landsat TM and ETM & Image classification and correlation analysis & $\begin{array}{l}\text { Maximum evaporation, } \\
\text { extreme conditions of soil } \\
\text { salinity, pronounced } \\
\text { difference between the dry } \\
\text { surface and wet zone } \\
\text { conditions }\end{array}$ & $\begin{array}{l}\text { (Alavi Panah et } \\
\text { al., 2008) }\end{array}$ \\
\hline 2008 & 2004-2006 & Colorado, USA & BSk (Cold Arid Steppe) & local $\left(500 \mathrm{~km}^{2}\right)$ & . & & & Ikonos,Landsat 5 & $\begin{array}{l}\text { Correlation analysis, ordinary least squares (OLS), spatial } \\
\text { autoregressive (spatial AR) modeling, and modif ed residual kriging } \\
\text { modeling }\end{array}$ & Saline river & $\begin{array}{l}\text { (Eldeiry \& } \\
\text { Garcia, 2008) }\end{array}$ \\
\hline 2007 & 1980 and 2000 & $\begin{array}{c}\text { Changling } \\
\text { County, China }\end{array}$ & Dwa & $\begin{array}{c}\text { local }(5728.4 \\
\left.\mathrm{km}^{2}\right)\end{array}$ & - & $\cdot$ & & Landsat TM & Cellular automata $(\mathrm{CA})$ model & Low precipitation & $\begin{array}{l}\text { (Xiaoxia et al., } \\
\text { 2007) }\end{array}$ \\
\hline 2007 & 2000 to 2005 & $\begin{array}{l}\text { San Luis Rio } \\
\text { Colorado Valley } \\
\text { (SLRCV) in } \\
\text { Sonora, Mexico }\end{array}$ & BWh (Hot Arid Desert) & local $\left(130 \mathrm{~km}^{2}\right)$ & . & . & . & $\begin{array}{l}\text { ASTER, Landsat (TM and } \\
\left.\text { ETM }{ }^{+}\right)\end{array}$ & Regression analysis, statistical analysis, correlation analysis & & $\begin{array}{c}\text { (Lobell \& } \\
\text { Ortiz- } \\
\text { monasterio, } \\
\text { 2007) }\end{array}$ \\
\hline 2007 & 2002-2003 & $\begin{array}{l}\text { Northwestern } \\
\text { China }\end{array}$ & BWk (Cold Arid Desert) & regional & . & . & & ASTER & $\begin{array}{l}\text { Spectral correlation mapper (SCM) algorithm } \\
\text { Regression analysis }\end{array}$ & $\begin{array}{l}\text { Rise in the groundwater } \\
\text { table }\end{array}$ & $\begin{array}{l}\text { (Brunner et al., } \\
\text { 2007) }\end{array}$ \\
\hline
\end{tabular}


Geographical coordinates of all selected case study areas associated with monitoring soil salinity via RS technology were plotted on the Köppen-Geiger Climate classification map as shown in Figure 4. Location of some case studies on the map is so close to each other and when the coordinates of those studies are shown in worldwide scale, they overlapped each other. The map illustrates that majority of the studies conducted in the north hemisphere between latitudes $10^{\circ} 00^{\prime} 0^{\prime} \mathrm{N}$ and $50^{\circ} 0^{\prime} 0^{\prime \prime} \mathrm{N}$. This belt includes the most arid and semi-arid regions of the world. The overview demonstrates that almost $63 \%$ of the selected case studies are located in arid and semi-arid regions of the world like Middle East countries, India, China, United States and some European countries where protecting lands from soil salinization and erosion are becoming a major concern for agricultural productivity. In fact, preserving agricultural lands and food supply for rapidly increasing population in these regions is highly troubled.

As shown in Table 1, the main reasons of soil salinization are investigated for each selected case study. Results indicate that both primary and secondary salinization can be considered as causes of soil salinity. Exploring studies which are performed in arid and semiarid regions of the world points out that high evapotranspiration and low precipitation rates together with the presence of minerals and parent material are the most common primary courses of soil salinization. Despite, in some cases which are located in the coastal regions, seawater intrusion is considered as another reason. Concerning secondary salinization, it is explored that poor agricultural irrigation practices and inappropriate drainage systems lead to accumulation of salts in the soil profile, and also cause rise of groundwater table.

\section{Conclusions and Recommendations}

Information on the climate regime and spatial extent of the selected case study areas, sensing approaches, and analysis and mapping methods were summarized to determine the current status of RS technology on detecting soil salinity in this review article. Ecological and economic importance of land has encouraged decision-makers of many countries to utilize $\mathrm{RS}$ for monitoring soil properties with the aim of optimizing sustainable land-use, boosting agricultural productivity, lessening drought effects, minimizing soil salinization and preventing soil erosion and compaction. Novelty of this study lies on the fact that geographical location of the selected case studies was plotted on Köppen-Geiger climate classification map for understanding the geographical location of these researches, and more importantly, for recognizing a relationship between soil salinity and climatic zones of case studies. The fact that $63 \%$ of the selected case studies are conducted in arid and semi-arid regions of the world indicates that soil salinization is becoming a major concern in many nations especially in such regions, and it is expected to affect countries of arid zones more vigorously and widely in the coming years if no protective measures are taken.

Further analysis and assessment at various levels extending from local to continental scale, are required to cope with the problem of soil salinity and its consequences. The most commonly used and recent RS mapping methods including support vector machine (SVM) method, random forest (RF) regression models, principle component analysis (PCA), partial least square regression (PLSR) analysis, multiple regression analysis, neural network model and various spectral soil salinity indices derived mostly from visible bands of electromagnetic spectrum were summarized. In addition to Landsat satellite systems, recently launched medium resolution satellites with multi-spectral data collection capability such as Chinese Huan Jing (HJ-1A), ESA Sentinel satellites being widely available have a strong potential to be used in soil salinity monitoring and mapping research with their temporal resolution capabilities.

\section{References}

Abbas, A., Khan, S., Hussain, N., Hanjra, M.A. \& Akbar, S. (2013). Characterizing soil salinity in irrigated agriculture using a remote sensing approach. Phys. Chem. Earth, 55(57), 43-52.

Abdellatif, D. (2017). Optical tool for salinity detection by remote sensing spectroscopy: application on Oran watershed. J. of Applied Remote Sensing, 11(3), 1-21.

Afrasinei, G.M., Melis, M.T., Buttau, C., Bradd, J.M., Arras, C. \& Ghiglieri, G. (2018). Assessment of remote sensing-based classification methods for change detection of salt-affected areas (Biskra area, Algeria). J. of Applied Remote Sensing, 11(1), 1-28.

Akramkhanov, A., Martius, C., Park, S.J. \& Hendrickx, J.M.H. (2011). Environmental factors of spatial distribution of soil salinity on flat irrigated terrain. Geoderma, 163, 55-62.

Akramkhanov, A. \& Vlek, P.L.G. (2012). The assessment of spatial distribution of soil salinity risk using neural network. Environ. Monit. Assess., 184, 2475-2485.

Alavi Panah, S.K., Goossens, R., Matinfar, H.R., Mohamadi, H., Ghadiri, M., Irannegad, H. \& Alikhah Asl, M. (2008). The efficiency of Landsat TM and $\mathrm{ETM}+$ thermal data for extracting soil information in arid regions. J. Agric. Sci. Technol., 10, 439-460.

Aldabaa, A.A.A., Weindorf, D.C., Chakraborty, S., Sharma, A. \& Li, B. (2015). Combination of proximal and remote sensing methods for rapid soil salinity quantification. Geoderma, 239-240, 34-46.

Aldakheel, Y.Y. (2011). Assessing NDVI spatial pattern as related to irrigation and soil salinity management in Al-Hassa Oasis, Saudi Arabia. J. Indian Soc. Remote Sens., 39, 171-180.

Alexakis, D.D., Daliakopoulos, I.N., Panagea, I.S. \& Tsanis, I.K. (2018). Assessing soil salinity using WorldView-2 multispectral images in Timpaki, Crete, Greece. Geocarto Int., 6049, 1-18. 
Allbed, A. \& Kumar, L. (2013). Soil salinity mapping and monitoring in arid and semi-arid regions using remote sensing technology: a review. Adv. Remote Sens., 2, 373-385.

Allbed, A., Kumar, L. \& Aldakheel, Y.Y. (2014a). Assessing soil salinity using soil salinity and vegetation indices derived from IKONOS highspatial resolution imageries: applications in a date palm dominated region. Geoderma, 230-231, 1-8.

Allbed, A., Kumar, L. \& Sinha, P. (2014b). Mapping and modelling spatial variation in soil salinity in the $\mathrm{Al}$ Hassa Oasis based on remote sensing indicators and regression techniques. Remote Sens., 6, 1137-1157.

An, D., Zhao, G., Chang, C., Wang, Z. \& Li, P. (2016). Hyperspectral field estimation and remote-sensing inversion of salt content in coastal saline soils of the Yellow River Delta. Int. J. Remote Sens., 37, 455470.

Arnous, M.O., El-Rayes, A.E. \& Green, D.R. (2015). Hydrosalinity and environmental land degradation assessment of the East Nile Delta region. Egypt. J. Coast. Conserv., 19, 491-513.

Arnous, M.O. \& Green, D.R. (2015). Monitoring and assessing waterlogged and salt-affected areas in the Eastern Nile Delta region, Egypt, using remotely sensed multi-temporal data and GIS. J. Coast. Conserv., 19, 369-391.

Arrouays, D., Lagacherie, P. \& Hartemink, A.E. (2017). Digital soil mapping across the globe. Geoderma Reg. 9, 1-4.

Bai, L., Wang, C., Zang, S., Zhang, Y., Hao, Q. \& Wu, Y. (2016). Remote sensing of soil alkalinity and salinity in the Wuyu'er-Shuangyang River Basin, Northeast China. Remote Sens., 8, 163.

Bannari, A. \& Guédon, A.M. (2016). Communications in soil science and plant analysis mapping slight and moderate saline soils in irrigated agricultural land using advanced land imager sensor (EO-1) data and semi-empirical models. Commun. Soil Sci. Plant Anal., 47, 1883-1906.

Bannari, A., Guedon, A.M., El-Harti, A., Cherkaoui, F.Z. \& El-Ghmari, A. (2008). Characterization of slightly and moderately saline and sodic soils in irrigated agricultural land using simulated data of advanced land imaging (EO-1) sensor. Commun. Soil Sci. Plant Anal. 39, 2795-2811.

Barut, İ. (2015). Origin of groundwater salinity and hydrogeochemical processes in a confined coastal karst aquifer: A cause of the Mandalia Bay (southeastern Aegean Sea coasts), International Journal of Environment and Geoinformatics (IJEGEO), 2 (1), 25-46.

Bhatt, M.J., Patel, A.D., Bhatti, P.M. \& Pandey, A.N. (2008). Effect of soil salinity on growth, water status and nutrient accumulation in seedlings of ziziphus mauritiana (RHAMNACEAE). Journal of Fruit and Ornamental Plant Research, 16, 383-401.

Bouaziz, M., Matschullat, J. \& Gloaguen, R. (2011). Improved remote sensing detection of soil salinity from a semi-arid climate in Northeast Brazil. Comptes Rendus-Geosci., 343, 795-803.

Brunner, P., Li, H.T., Kinzelbach, W. \& Li, W.P. (2007). Generating soil electrical conductivity maps at regional level by integrating measurements on the ground and remote sensing data. Int. J. Remote Sens., 28, 3341-3361.

Büyüksalih, İ. \& Gazioğlu, C. (2019). New Approach in Integrated Basin Modelling: Melen Airborne LIDAR, International Journal of Environment and Geoinformatics (IJEGEO), 6(1): 22-32.

Carol, E.S., Kruse, E.E. \& Cellone, F.A. (2015). Salinization of soils in marshes. Case study: Humedal of Samborombón Bay, (Argentina Salinización de suelos en marismas. Caso de estudio: Humedal de la Bahía Samborombón, Argentina). Rev. la Fac. Ciencias Agrar., 47, 97-107.

Casterad, A. (2018). Assessment of soil salinity during the first years of transition from flood to sprinkler irrigation. Sensors., 18, 616.

Chen, D. \& Chen, H.W. (2013). Using the Köppen classi fi cation to quantify climate variation and change: An example for 1901-2010. Environmental Development, 6, 69-79.

Chuangye, S., Hongxu, R.E.N. \& Chong, H. (2016). Estimating soil salinity in the Yellow River Delta, Eastern China - an integrated approach using spectral and terrain indices with the generalized additive model. Pedosph. An Int. J., 26, 626-635.

Daliakopoulos, I.N., Tsanis, I.K., Koutroulis, A., Kourgialas, N.N., Varouchakis, A.E., Karatzas, G.P. \& Ritsema, C.J. (2016). The threat of soil salinity: A European scale review. Sci. Total Environ., 573, 727-739.

Das, S., Choudhury, M.R. \& Das, S. (2016). Earth observation and geospatial techniques for soil salinity and land capability Assessment over Sundarban Bay of Bengal Coast, India. Geodesy and Cartography. $65,163-192$.

Ding, J. L., Wu, C. M. \& Tiyip, T. (2011). Study on Soil Salinization Information in Arid Region Using Remote Sensing Technique. Agric. Sci. China, 10, 404-411.

Ding, J. \& Yu, D. (2014). Monitoring and evaluating spatial variability of soil salinity in dry and wet seasons in the Werigan-Kuqa Oasis, China, using remote sensing and electromagnetic induction instruments. Geoderma, 235-236, 316-322.

Dutkiewicz, A., Lewis, M. \& Ostendorf, B. (2009). Evaluation and comparison of hyperspectral imagery for mapping surface symptoms of dryland salinity. Int. J. Remote Sens., 30, 693-719.

Dutkiewicz, A. (2006). Evaluating hyperspectral imagery for mapping surface symptoms of dryland salinity with hyperspectral imagery. (Ph.D Thesis). Discipline of Soil and Land Systems School of Earth and Environmental Sciences the University of Adelaide, Australia.

Ekercin, S. \& Örmeci, C. (2008). Estimating soil salinity using satellite remote sensing data and real-time field sampling. Environmental Engineering Science, 25(7), 981-988.

El, A., Lhissou, R., Chokmani, K., Ouzemou, J., Hassouna, M., Mostafa, E. \& El, A. (2016). Spatiotemporal monitoring of soil salinization in irrigated Tadla Plain (Morocco) using satellite 
spectral indices. Int. J. Appl. Earth Obs. Geoinf., 50, 64-73.

Eldeiry, A.A. \& Garcia, L.A. (2008). Detecting soil salinity in alfalfa fields using spatial modeling and remote sensing. Soil Sci. Soc. Am. J., 72, 201-211.

Eldeiry, A.A., Garcia, L.A. \& Reich, R.M. (2008). Soil salinity sampling strategy using spatial modeling techniques, remote sensing, and field data. J. Irrig. Drain. Eng., 134, 768-777.

Elhag, M. \& Bahrawi, J.A. (2017). Soil salinity mapping and hydrological drought indices assessment in arid environments based on remote sensing techniques. Geosci. Instrum. Method. Data Syst., 6, 149-158.

Elhag, M. (2016). Evaluation of different soil salinity mapping using remote sensing techniques in arid ecosystems, Saudi Arabia. Journal of Sensors, 8, Article ID 7596175

Elnaggar, A. A. \& Noller, J.S. (2009). Application of remote-sensing data and decision-tree analysis to mapping salt-affected soils over large areas. Remote Sens., 2, 151-165.

Fallah Shamsi, S.R., Zare, S. \& Abtahi, S.A. (2013). Soil salinity characteristics using moderate resolution imaging spectroradiometer (MODIS) images and statistical analysis. Arch. Agron. Soil Sci., 59, 471489.

Esetlili, MT., Bektaş Balçık, F., Balık Şanlı, f., Üstüner, M., Kalkan, K., Göksel, Ç., Gazioğlu, C. \& Kurucu, Y. (2018). Comparison of Object and Pixel-Based Classifications For Mapping Crops Using Rapideye Imagery: A Case Study Of Menemen Plain, Turkey, International Journal of Environment and Geoinformatics (IJEGEO), 5(2), 231-243.

Fan, X., Liu, Y., Tao, J. \& Weng, Y. (2015). Soil salinity retrieval from advanced multi-spectral sensor with partial least square regression. Remote Sens., 7, 488511.

Fan, X., Pedroli, B., Liu, G., Liu, Q., Liu, H. \& Shu, L. (2012). Soil salinity development in the yellow river delta in relation to groundwater dynamics. Land Deg. and Development., 23, 175-189.

Fan, X., Weng, Y. \& Tao, J. (2016). Towards decadal soil salinity mapping using Landsat time series data. Int. J. Appl. Earth Obs. Geoinf., 52, 32-41.

Gazioğlu, C. (2018). Biodiversity, Coastal Protection, Promotion and Applicability Investigation of the Ocean Health Index for Turkish Seas, International Journal of Environment and Geoinformatics (IJEGEO). 5 (3), 353-367.

Gazioğlu, C. Burak, S.Z., Alpar, B., Türker, A. \& Barut I.F. (2010). Foreseeable impacts of sea level rise on the southern coast of the Marmara Sea (Turkey), Water Policy, 12(6), 932-943.

Goldshleger, N., Ben-Dor, E., Lugassi, R. \& Eshel, G. (2010). Soil degradation monitoring by remote sensing: examples with three degradation processes. Soil Sci. Soc. Am. J., 74, 1433-1445.

Goldshleger, N., Livne, I., Chudnovsky, A. \& Ben-Dor, E. (2012). New results in integrating passive and active remote sensing methods to assess soil salinity: a case study from Jezre'el Valley, Israel. Soil Science, 177(6), 392-401.
Gorji, T., Alganci, U., Sertel, E. \& Tanik, A. (2018). Comparing two different spatial interpolation approaches to characterize spatial variability of soil properties in Tuz Lake Basin - Turkey, Conference: 19th International Symposium on Environmental Pollution and its Impact on Life in the Mediterranean Region (MESAEP), At Rome, Italy.

Gorji, T., Sertel, E. \& Tanik, A. (2017a). Monitoring soil salinity via remote sensing technology under data scarce conditions: A case study from Turkey. Ecol. Indic., 74, 384-391.

Gorji, T., Sertel, E. \& Tanik, A. (2017b). Recent Satellite Technologies for Soil Salinity Assessment with Special Focus on Mediterranean Countries. Fresenius Environmental Bulletin Journal, 26(1), 196-203.

Gorji, T., Tanik, A. \& Sertel, E. (2015). Soil Salinity Prediction, Monitoring and Mapping Using Modern Technologies. Procedia Earth and Planetary Science, 15, $507-512$.

Goto, K., Goto, T., Nmor, J.C., Minematsu, K. \& Gotoh, K. (2014). Evaluating salinity damage to crops through satellite data analysis: application to typhoon affected areas of southern Japan. Nat. Hazards, 75, 2815-2828.

Grunwald, S., Vasquesy, G.M. \& Rivero, R.G. (2015). Fusion of soil and remote sensing data to model soil properties. Advances in Agronomy, 131, 1-109.

Guo, Y., Shi, Z., Zhou, L. Qing, Jin, X., Tian, Y. Feng, Teng. \& Fen, H. (2013). Integrating remote sensing and proximal sensors for the detection of soil moisture and salinity variability in coastal areas. $J$. Integr. Agric., 12, 723-731.

Guo, Y., Shi, Z., Li, H.Y. \& Triantafilist, J. (2013). Application of digital soil mapping methods for identifying salinity management classes based on a study on coastal central China. Soil Use and Management, 29, 445-456.

Gutierrez, M.\& Johnson, E. (2010). Temporal variations of natural soil salinity in an arid environment using satellite images. J. South Am. Earth Sci., 30, 46-57.

Hamzeh, S., Naseri, A. A., AlaviPanah, S.K., Mojaradi, B., Bartholomeus, H.M., Clevers, J.G.P.W. \& Behzad, M. (2013). Estimating salinity stress in sugarcane fields with spaceborne hyperspectral vegetation indices. Int. J. Appl. Earth Obs. Geoinf., 21, 282-290.

Hereher, M.E. \& Ismael, H. (2016). The application of remote sensing data to diagnose soil degradation in the Dakhla depression-Western Desert, Egypt. Geocarto Int., 31, 527-543.

Iqbal, F. (2011). Detection of salt-affected soil in ricewheat area using satellite image. African J. Agric. Res., 6, 4973-4982.

Ivits, E., Cherlet, M., Tóth, T., Ska, K.E.L.Ń. \& Tóth, G. (2013). Characterisation of productivity limitation of salt-affected lands in different climatic regions of Europe using remote sensing derived productivity indicators. Land Deg. and Development., 24, 438452.

Ivushkin, K., Bartholomeus, H., Bregt, A.K. \& Pulatov, A. (2017). Satellite thermography for soil salinity 
assesment of cropped areas in Uzbekistan. Land Deg. and Development., 28, 870-877.

İmamoğlu, MZ. \& Sertel, E. (2016). Analysis of Different Interpolation Methods for Soil Moisture Mapping Using Field Measurements and Remotely Sensed Data. International Journal of Environment and Geoinformatics (IJEGEO), 3(3), 11-25.

Jabbar, M.T. \& Zhou, J. (2012). Assessment of soil salinity risk on the agricultural area in Basrah Province, Iraq: Using remote sensing and GIS techniques. J. Earth Sci., 23, 881-891.

Jacobus, S. \& Niekerk, A.V. (2016a). Identification of WorldView-2 spectral and spatial factors in detecting salt accumulation in cultivated fields. Geoderma, $273,1-11$.

Jacobus, S. \& Niekerk, A.V. (2016b). An evaluation of supervised classifiers for indirectly detecting saltaffected areas at irrigation scheme level. Int. J. Appl. Earth Obs. Geoinf., 49, 138-150.

Jiang, H. \& Xu, J. (2018). Estimating soil salt components and salinity using hyperspectral remote sensing data in an arid area of China. Journal of Applied Remote Sensing, 11, 016043-1.

Jin, P., Li, P., Wang, Q. \& Pu, Z. (2015). Developing and applying novel spectral feature parameters for classifying soil salt types in arid land. Ecol. Indic., $54,116-123$.

Jin, X.M., Vekerdy, Z., Zhang, Y.K. \& Liu, J.T. (2012). Soil salt content and its relationship with crops and groundwater depth in the Yinchuan Plain (China) using remote sensing. Arid L. Res. Manag., 26, 227235.

Judkins, G. \& Myint, S. (2012). Spatial variation of soil salinity in the Mexicali Valley, Mexico: application of a practical method for agricultural monitoring. Environ. Manage., 50, 478-489.

Justin, G.K. \& Suresh, K. (2015). Hyperspectral remote sensing in characterizing soil salinity severity using SVM technique-a case study of alluvial plains. Int. J. Adv. Remote Sens. and GIS, 4, 1344-1360.

Kaya, H., Erginal, G., Çakır, Ç. Gazioğlu, C. \& Erginal, E. (2017). Ecological risk evaluation of sediment core samples, Lake Tortum (Erzurum, NE Turkey) using environmental indices, International Journal of Environment and Geoinformatics (IJEGEO), 4 (3), 227-239.

Kaya, Ş., Çelik, B., Gazioğlu, C. Algancı, U. \& Şeker, DZ. (2017). Assessment of the Relationship between Land Cover and Land Surface Temperatures Utilizing Remotely Sensed Data: A Case Study of Silivri, 19th MESAEP Symposium on Environmental and Health Inequity., Roma, ITALYA, 3-6 Dec 2017.

Kobryn, H.T., Lantzke, R., Bell, R. \& Admiraal, R. (2015). Remote sensing for assessing the zone of benefit where deep drains improve productivity of land affected by shallow saline groundwater. $J$. Environ. Manage., 150, 138-148.

Laiskhanov, S.U., Otarov, A., Savin, I.Y., Tanirbergenov, S.I., Mamutov, Z.U., Duisekov, S.N. \& Zhogolev, A. (2016). Dynamics of soil salinity in irrigation areas in South Kazakhstan. Polish J. Env. Studies, 25, 2469-2475.
Liu, L., Ji, M. \& Buchroithner, M. (2018). A case study of the forced invariance approach for soil salinity estimation in vegetation-covered terrain using airborne hyperspectral imagery. Int. J. Geo-Inf, 7(2), 48.

Lobell, D.B., Lesch, S.M., Corwin, D.L., Ulmer, M.G., Anderson, K.A., Potts, D.J., Doolittle, J.A., Matos, M.R. \& Baltes, M.J. (2010). Regional-scale assessment of soil salinity in the Red River Valley using multi-year MODIS EVI and NDVI. J. Environ. Qual., 39, 35-41.

Lobell, D.B. \& Ortiz-monasterio, J.I. (2007). Identification of saline soils with multiyear remote sensing of crop yields. Soil Science Society of America Journal, 71, 777-783.

Ma, L., Ma, F., Li, J., Gu, Q., Yang, S., Wu, D., Feng, J. \& Ding, J. (2017). Characterizing and modeling regional-scale variations in soil salinity in the arid oasis of Tarim Basin, China. Goederma, 305, 1-11.

Ma, L. \& Yang, S. (2018). Modeling variations in soil salinity in the oasis of Junggar. Land Degr. and Development, 29(3), 551-562.

Manchanda, M.L., Kudrat, M. \& Tiwari, A.K. (2002). Soil survey and mapping using remote sensing. Trop. Ecol., 43, 61-74.

Mandal, A.K. \& Sharma, R.C. (2011). Delineation and Characterization of Waterlogged Salt-affected Soils in IGNP Using Remote Sensing and GIS. J. Indian Soc. Remote Sens. 39, 39-50.

Matinfar, H.R., Alavi Panah, S.K., Zand, F. \& Khodaei, K. (2013). Detection of soil salinity changes and mapping land cover types based upon remotely sensed data. Arab. J. Geosci., 6, 913-919.

Mayak, S., Tirosh, T. \& Glick, B.R. (2004). Plant growth-promoting bacteria confer resistance in tomato plants to salt stress. Plant Physiol. Biochem., 42, 565-572.

Meimei, Z. \& Ping, W. (2011). Using HJ - I satellite remote sensing data to surveying the saline soil distribution in Yinchuan Plain of China. African $J$. Agric. Reseearch, 6, 6592-6597.

Melendez-Pastor, I., Navarro-Pedreño, J., Koch, M. \& Gómez, I. (2010). Applying imaging spectroscopy techniques to map saline soils with ASTER images. Geoderma, 158, 55-65.

Meng, L., Zhou, S., Zhang, H., Bi, X. (2016). Estimating soil salinity in different landscapes of the Yellow River Delta through Landsat OLI / TIRS and ETM + Data. J. Coast. Conserv., 20(4), 271-279.

Metternicht, G.I. \& Zinck, J.A. (2003). Remote sensing of soil salinity: potentials and constraints. Remote Sens. Environ., 85, 1-20.

Mitran, T., Ravisankar, T., Fyzee, M.A., Suresh, J.R., Sujatha, G. \& Sreenivas, K. (2015). Retrieval of soil physicochemical properties towards assessing saltaffected soils using Hyperspectral Data. Geocarto Int., 30, 701-721.

Moreira, L.C.J., Teixeira, A.D.S. \& Galvão, L.S. (2015). Potential of multispectral and hyperspectral data to detect saline-exposed soils in Brazil. GIScience Remote Sens., 52, 416-436.

Morshed, M., Islam, T. \& Jamil, R. (2016). Soil salinity detection from satellite image analysis: an integrated 
approach of salinity indices and field data. Envi. Monitoring and Assessment, 188(119), 2-10.

Mulder, V.L., Bruin, S. De, Schaepman, M.E. 6 Mayr, T.R. (2011). The use of remote sensing in soil and terrain mapping A review. Geoderma, 162, 1-19.

Nawar, S., Buddenbaum, H., Hill, J. \& Kozak, J. (2014). Modeling and mapping of soil salinity with reflectance spectroscopy and landsat data using two quantitative methods (PLSR and MARS). Remote Sens., 6, 10813-10834.

Nurmemet, I., Ghulam, A., Tiyip, T., Elkadiri, R., Ding, J.L., Maimaitiyiming, M., Abliz, A., Sawut, M., Zhang, F., Abliz, A. \& Sun, Q. (2015). Monitoring soil salinization in Keriya River Basin, Northwestern China using passive reflective and active microwave remote sensing data. Remote Sens., 7, 8803-8829.

Odeh, I.O.A. \& Onus, A. (2008). Spatial analysis of soil salinity and soil structural stability in a semi-arid region of New South Wales, Australia. Environ. Manage., 42, 265-278.

Pang, G., Wang, T., Liao, J. \& Li, S. (2014). Quantitative model based on field-derived spectral characteristics to estimate soil salinity in Minqin County, China. Soil Sci. Soc. Am. J., 78(2), 546-555.

Periasamy, S. \& Shanmugam, R.S. (2017). Multispectral and microwave remote sensing models to survey. Land Deg. and Development, 28(4), 1412-1425.

Phonphan, W., Tripathi, N.K., Tipdecho, T. \& Eiumnoh, A. (2014). Modelling electrical conductivity of soil from backscattering coefficient of microwave remotely sensed data using artificial neural network. Geocarto Int., 29, 842-859.

Quan, Q., Shen, B., Xie, J.C., Luo, W. \& Wang, W.Y. (2013). Assessing soil salinity in the fields of western China using spatial modeling and remote sensing. Acta Agric. Scand. Sect. B-Soil Plant Sci., 63, 289296.

Rahmati, M. \& Hamzehpour, N. (2017). Quantitative remote sensing of soil electrical conductivity using $\mathrm{ETM}+$ and ground measured data. Int. J. Remote Sens., 38, 123-140.

Rukhovich, D.I., Pankova, E.I., Chernousenko, G.I. \& Koroleva, P. V. (2010). Long-term salinization dynamics in irrigated soils of the Golodnaya Steppe and methods of their assessment on the basis of remote sensing data. Eurasian Soil Sci., 43, 682-692.

Satır, O. \& Berberoğlu, S. (2016). Crop yield prediction under soil salinity using satellite derived vegetation indices. Field Crop. Res., 192, 134-143.

Saghafi, M. (2017). Application of remote sensing indices for mapping salt-affected areas by using field data methods. International Journal of Advanced and Applied Sciences, 4, 181-187.

Scudiero, E., Corwin, D.L., Anderson, R.G., Yemoto, K., Clary, W., Luke, Z. \& Todd, W. (2017). Remote sensing is a viable tool for mapping soil salinity in agricultural lands. California Agriculture, 71(4), 231238.

Scudiero, E., Skaggs, T.H. \& Corwin, D.L. (2015). Regional-scale soil salinity assessment using Landsat ETM+ canopy reflectance. Remote Sens. Environ., 169, 335-343.
Setia, R., Lewis, M., Marschner, P., Raja Segaran, R., Summers, D. \& Chittleborough, D. (2013). Severity of salinity accurately detected and classified on a paddock scale with high resolution multispectral satellite imagery. Land Deg. and Development., 24, 375-384.

Shrestha, D.P. \& Farshad, A. (2009). Mapping salinity hazard: an integrated application of remote sensing and modeling-based techniques, Chapter 13. In: Remote Sensing of Soil Salinization: Impact on Land Management (p. 257-272). NY, USA: CRC.

Sidike, A., Zhao, S. \& Wen, Y. (2014). Estimating soil salinity in Pingluo County of China using QuickBird data and soil reflectance spectra. Int. J. Appl. Earth Obs. Geoinf., 26, 156-175.

Şekertekin, A., Marangoz, AM. \& Abdikan, S. (2018). Soil Moisture Mapping Using Sentinel-1A Synthetic Aperture Radar Data. International Journal of Environment and Geoinformatics (IJEGEO), 5(2), 178-188.

Triki Fourati, H., Bouaziz, M., Benzina, M. \& Bouaziz, S. (2015). Modeling of soil salinity within a semiarid region using spectral analysis. Arab. J. Geosci., 8(12), 11175-11182.

Ülker, D., Ergüven, O. \& Gazioğlu, c. (2018). Socioeconomic impacts in a Changing Climate: Case Study Syria. International Journal of Environment and Geoinformatics (IJEGEO), 5(1), 84-93.

Vermeulen, D. \& Niekerk, A.V. (2016). Evaluation of a WorldView-2 image for soil salinity monitoring in a moderately affected irrigated area. J. Appl. Remote Sens., 10(2), 026025.

Vermeulen, D. \& Niekerk, A.V. (2017). Geoderma Machine learning performance for predicting soil salinity using different combinations of geomorphometric covariates. Geoderma, 299, 1-12.

Wang, F., Chen, X., Luo, G.P., Ding, J.L. \& Chen, X.F. (2013). Detecting soil salinity with arid fraction integrated index and salinity index in feature space using Landsat TM imagery. J. Arid Land, 5, 340353.

Wang, X., Zhang, F., Ding, J., Kung, H., Latif, A. \& Johnson, V.C. (2018). Estimation of soil salt content (SSC) in the Ebinur Lake Wetland National Nature Reserve (ELWNNR), Northwest China, based on a Bootstrap-BP neural network model and optimal spectral indices. Sci. Total Environ., 615, 918-930.

Weng, Y.L., Gong, P. \& Zhu, Z.L. (2010). A Spectral Index for Estimating Soil Salinity in the Yellow River Delta Region of China Using EO-1 Hyperion Data. Pedosphere, 20, 378-388.

Wu, J., Vincent, B., Yang, J., Bouarfa, S. \& Vidal, A. (2008). Remote sensing monitoring of changes in soil salinity: a case study in inner Mongolia, China. Sensors, 8, 7035-7049.

Wu, W., Al-shafie, W.M., Mhaimeed, A.S., Ziadat, F., Nangia, V. \& Payne, W.B. (2014). Soil salinity mapping by multiscale remote sensing in Mesopotamia, Iraq. IEEE Journal of Selected Topics in Applied Earth Observations and Remote Sensing, 7(11), 4442-4452.

Xiaoxia, S., Yunhao, C., Jianwei, Y., Jing, L. \& Cheng, P. (2007). Simulating and forecasting soil- 
salinisation evolution: A case study on Changling County, Jilin province, China. New Zeal. J. Agric. Res., 50, 975-981.

Yahiaoui, I., Douaoui, A., Zhang, Q. \& Ziane, A. (2015). Soil salinity prediction in the Lower Cheliff plain (Algeria) based on remote sensing and topographic feature analysis. J. Arid Land, 7, 794-805.

Ya-kun, W., Jin-song, Y., \& Xiao-Ming, L. (2009). Study on spatial variability of soil salinity based on spectral indices and EM38 readings. Spectroscopy and Spectral Analysis, 29(4), 1023-1027.

Yong-hua, Q., Xiao- liang, D., Hong-yong, G., Aiping, C., Yong-qing, A. Jin- ling, S., Hongm I. \& Tao, H. (2009). Quantitative retrieval of soil salinity using hyperspectral data in the region of Inner Mongolia hetao irrigation district. Spectroscopy and Spectral Analysis, 29(5), 1362-1366.
Yu, R., Liu, T., Xu, Y., Zhu, C., Zhang, Q., Qu, Z., Liu, X. \& Li, C. (2010). Analysis of salinization dynamics by remote sensing in Hetao Irrigation District of North China. Agric. Water Manag., 97, 1952-1960.

Zeng, W.,Zhang, D., Fang,Y.Wu, J. \& Huang, J. (2018). Comparison of partial least square regression, support vector machine, and deep-learning techniques for estimating soil salinity from hyperspectral data. J. Appl. Remote Sens., 12(2), 022204.

Zhang, T.-T., Qi, J.-G., Gao, Y., Ouyang, Z.-T., Zeng, S.-L. \& Zhao, B. (2015). Detecting soil salinity with MODIS time series VI data. Ecol. Indic., 52, 480489.

Zinck, J.A. \& Metternicht, G. (2009). Soil salinity and salinization hazard, Chapter 1. In: Remote Sensing of Soil Salinization: Impact on Land Management. (p. 3-60). NY, USA: CRC. 